\title{
Test of the Goldstone-boson approximation with fermions
}

\author{
Loyal Durand* \\ Department of Physics, University of Wisconsin-Madison, 1150 University Avenue, Madison, Wisconsin 53706 \\ Kurt Riesselmann ${ }^{\dagger}$ \\ Physik Department T30, Technische Universität München, James-Franck Strasse, 85747 Garching b. München, Germany
}

(Received 5 December 1995; revised manuscript received 11 September 1996)

\begin{abstract}
The calculation of the leading electroweak corrections to physical transition matrix elements in powers of $M_{H}^{2} / v^{2}$ can be greatly simplified in the limit $M_{H}^{2} \gg M_{W}^{2}, M_{Z}^{2}$ by the use of the Goldstone-boson approximation. In this approximation, the vector bosons $W^{ \pm}$and $Z$ are replaced by the associated scalar Goldstone bosons $w^{ \pm}, z$ which appear in the symmetry-breaking sector of the standard model in the limit of vanishing gauge couplings. The approximation remains valid in the presence of the Yukawa interactions between the fermions and the Higgs and Goldstone bosons provided the renormalization conditions are formulated so as to be consistent with the standard model. As a concrete test of the approximation scheme, we calculate the dominant radiative corrections to fermionic Higgs-boson decays at one loop including the virtual effects of a heavy top quark. We apply the result to the decays $H \rightarrow t \bar{t}$ and $H \rightarrow b \bar{b}$, and find that the results including fermions are quite accurate numerically for Higgs-boson masses $M_{H}>400$ (350) GeV, respectively, even for $m_{t}=175 \mathrm{GeV}$. We discuss the associated renormalization problem to all orders in an appendix to the paper. [S0556-2821(97)00503-1]
\end{abstract}

PACS number(s): 12.15.Lk, 11.15.Bt, 14.80.Bn

\section{INTRODUCTION}

The investigation of the physics of the Higgs sector of electroweak theory is one of the most important problems for high-energy experiments in the near future. Radiative corrections to high-energy processes may become very important in the process of distinguishing the standard model (SM) Higgs sector from other theoretical models. Moreover, the effects of a Higgs boson on low-energy observables such as the electroweak $\rho$ parameter already need to be included in comparing the theory to present precision measurements. However, the calculation of the complete higher-order corrections is a difficult task. It is therefore important if one can calculate the most significant corrections relatively simply within a well-defined approximation. In the case of a heavy Higgs boson, such an approach is provided by the Goldstone-boson approximation (GBA). This is closely related to the original Goldstone-boson equivalence theorem $[1,2]$, but deals with internal rather than external gauge bosons. In particular, it can be shown through powercounting arguments $[3,4]$ that the leading correction to a physical process in powers of $M_{H}^{2} / v^{2}$ can be obtained at any order by replacing the vector bosons $W^{ \pm}$and $Z$ of the SM by the associated scalar Goldstone bosons $w^{ \pm}, z$ which appear in the symmetry-breaking sector of the theory in the limit of vanishing gauge couplings. This replacement of vector by scalar bosons results in a substantial simplification of the calculations. In the approach described here, we work with the reduced theory from the beginning.

Previous calculations based on the Goldstone-boson approximation have mostly been concerned with processes in

\footnotetext{
*Electronic address: 1durand@wishep.physics.wisc.edu

${ }^{\dagger}$ Electronic address: kurtr@physik.tu-muenchen.de
}

which both the external and internal particles are Higgs bosons $H$ or longitudinally polarized gauge bosons $W_{L}$ and $Z_{L}[1,2,5-12]$, and have neglected corrections from internal fermion loops. An exception is the work of Barbieri et al. [13], who calculated the $m_{t}^{4}$ corrections to the decays $Z \rightarrow \mu^{+} \mu^{-}$and $Z \rightarrow b \bar{b}$ to one loop. The existence of a heavy top quark leads to a large Yukawa coupling $g_{t}$ in the SM. In the case of a not-so-heavy Higgs boson, one can expect the radiative corrections which involve $g_{t}$ to be comparable in magnitude to the corrections which involve the quartic Higgs coupling $\lambda=M_{H}^{2} / 2 v^{2}$. These contributions can again be taken into account in a simple way using the Goldstoneboson approximation: In the limit of vanishing gauge couplings, the fermions couple only to the Higgs boson and the Goldstone bosons in the symmetry-breaking sector of the theory.

While the basis of the Goldstone-boson approximation is clear physically as resulting from the smooth limit of the SM as the gauge couplings are taken to zero, its implementation starting from the scalar theory with fermions is not completely straightforward. In particular, the requirement that it reproduce the leading corrections in the SM severely restricts the choice of renormalization scheme and the definitions of the couplings. We discuss this problem in detail in an appendix to this paper, and present a framework valid to all orders in $\lambda$ and $g_{f}$ for vanishing gauge couplings, results which will be useful in future calculations.

The Goldstone-boson approximation is known to be valid formally for $M_{H}$ sufficiently large compared to $M_{W}, M_{Z}$. An important question, however, is how heavy the Higgs boson must be before the GBA gives a quantitatively good approximation to the full electroweak theory. We study that question and illustrate our method here at the one-loop level using the fermionic decay of the Higgs boson, $H \rightarrow f \bar{f}$. We find for the 
observed top-quark mass $m_{t} \approx 175 \mathrm{GeV}[14]$ that the GBA result approximates the full electroweak correction to $\Gamma(H \rightarrow t \bar{t})$ very well for $M_{H}>400 \mathrm{GeV}$, but fails near the threshold at $350 \mathrm{GeV}$ where the effects of the gauge interactions become important. The result for $\Gamma(H \rightarrow b \bar{b})$ is also quite good for $M_{H}>350 \mathrm{GeV}$, though the accuracy is reduced by a cancellation between the Yukawa and Higgsboson contributions. The gauge couplings give the dominant, very small, corrections for lower values of $M_{H}$.

\section{LAGRANGIAN AND RENORMALIZATION SCHEME}

\section{A. Framework}

We will be concerned in later sections with the calculation of the leading contributions to the decay rate of the Higgs boson to fermions, $H \rightarrow f \bar{f}$, in the limit of large Higgsboson and top-quark masses. These contributions can be calculated rather simply using the Goldstone-boson approximation $[1,2,15,16,3,4]$. This approximation corresponds physically to the limit of vanishing gauge couplings, $g, g^{\prime} \rightarrow 0$, and the consequent decoupling of the tranverse gauge bosons from the remainder of the standard electroweak model. The remnant theory is defined by the Lagrangian $\mathcal{L}_{\mathrm{GBA}}=\mathcal{L}_{H}+\mathcal{L}_{F}$, where $\mathcal{L}_{H}$ is the Lagrangian for the scalar fields in the Higgs- or symmetry-breaking sector (the would-be Goldstone bosons of the full $\mathrm{SM}$ ), and $\mathcal{L}_{F}$ is the Lagrangian for the fermions which includes their Yukawa interactions with the scalar fields. If the couplings and renormalizations are properly defined, the reduced theory is supposed to give a good approximation to the full electroweak theory for $M_{H}$ sufficiently large compared to $M_{W}$. The result in our case is an expansion for the dominant contributions to $\Gamma(H \rightarrow f \bar{f})$ in powers of the quartic Higgs coupling $\lambda=M_{H}^{2} / 2 v^{2}=G_{F} M_{H}^{2} / \sqrt{2}$ and the top-quark Yukawa coupling $g_{t}=\sqrt{2} m_{t} / v$. Corrections associated with the transverse gauge bosons are suppressed by powers of the small gauge couplings $g, g^{\prime}$.

We will work entirely with the reduced theory using the Lagrangian

$$
\mathcal{L}_{\mathrm{GBA}}=\mathcal{L}_{H}^{0}+\mathcal{L}_{F}^{0}+\text { counterterms },
$$

where

$$
\begin{aligned}
& \mathcal{L}_{H}^{0}=\frac{1}{2}\left(\partial_{\mu} \Phi\right)^{\dagger}\left(\partial^{\mu} \Phi\right)-\frac{1}{4} \lambda\left(\Phi^{\dagger} \Phi\right)^{2}+\frac{1}{2} \mu^{2} \Phi^{\dagger} \Phi \\
\mathcal{L}_{F}^{0}= & i \bar{\Psi}_{L} b \Psi_{L}+i \bar{\psi}_{t, R} \partial \psi_{t, R}+i \bar{\psi}_{b, R} \partial \psi_{b, R}-\frac{1}{\sqrt{2}} g_{t} \bar{\Psi}_{L} \widetilde{\Phi} \psi_{t, R} \\
& -\frac{1}{\sqrt{2}} g_{b} \bar{\Psi}_{L} \Phi \psi_{b, R}+\text { H.c. }+\cdots
\end{aligned}
$$

We have written only the top- and bottom-quark contributions to $\mathcal{L}_{F}^{0}$; the remaining fermionic contributions have the same form, with no right-handed contributions for the neutrinos. In these expressions, $\Psi_{L}, \Phi$, and $\widetilde{\Phi}$ are $\mathrm{SU}(2)_{L}$ doublets with normalizations defined by

$$
\begin{gathered}
\Psi_{L}=\left(\begin{array}{c}
\psi_{t, L} \\
\psi_{b, L}
\end{array}\right), \quad \psi_{f, L}=\frac{1}{2}\left(1-\gamma^{5}\right) \psi_{f}, \\
\Phi=\left(\begin{array}{c}
w_{1}+i w_{2} \\
h+i z
\end{array}\right)=\left(\begin{array}{c}
\sqrt{2} w^{+} \\
h+i z
\end{array}\right), \\
\widetilde{\Phi}=i \sigma_{2} \Phi^{*}=\left(\begin{array}{c}
h-i z \\
-w_{1}+i w_{2}
\end{array}\right)=\left(\begin{array}{c}
h-i z \\
-\sqrt{2} w^{-}
\end{array}\right) .
\end{gathered}
$$

The right-handed fields are $\mathrm{SU}(2)_{L}$ singlets, with

$$
\psi_{f, R}=\frac{1}{2}\left(1+\gamma^{5}\right) \psi_{f} .
$$

The expressions in Eqs. (2.2) and (2.3) include all possible $\mathrm{SU}(2)_{L} \times \mathrm{U}(1)_{Y}$-symmetric terms consistent with renormalizability of the Lagrangian. The counterterms necessary to effect the renormalization without breaking the symmetry must have the same forms and can be introduced by independent multiplicative rescalings of each term above. Thus, in the case of the Higgs Lagrangian, all possible symmetric counterterms are generated by multiplying the kinetic energy term by a factor $Z_{\Phi}$, replacing $\lambda$ by $\lambda+\delta \lambda$, and $\mu^{2}$ by $\mu^{2}+\delta \mu^{2}$. Because the minimum in the Higgs potential is at a nonzero value of $\Phi^{\dagger} \Phi$ for $\mu^{2}>0, \Phi$ has a nonzero expectation value $v$ in the physical vacuum. We will display this by rewriting the field $h$ as $h=H+v$ and expanding around the physical vacuum in which $\langle\Omega|H| \Omega\rangle=\langle\Omega|\mathbf{w}| \Omega\rangle=0$. The renormalized Higgs Lagrangian then has the form

$$
\begin{aligned}
\mathcal{L}_{H}= & \frac{1}{2} Z_{\Phi}\left(\partial_{\mu} \mathbf{w} \cdot \partial^{\mu} \mathbf{w}+\partial_{\mu} H \partial^{\mu} H\right)-\frac{1}{4}(\lambda+\delta \lambda) \\
& \times\left(\mathbf{w}^{2}+H^{2}+2 v H\right)^{2}-\frac{1}{2}\left[(\lambda+\delta \lambda) v^{2}-\mu^{2}-\delta \mu^{2}\right] \\
& \times\left(\mathbf{w}^{2}+H^{2}+2 v H\right),
\end{aligned}
$$

where $\mathbf{w}$ is the $\mathrm{SO}(3)$ vector $\left(w_{1}, w_{2}, w_{3}\right)$ of Goldstone bosons with $w_{3}=z$, and we have dropped an additive constant.

It is convenient for calculation to define a set of rescaled or "bare" fields $H_{0}, \mathbf{w}_{0}$ and the corresponding $\mathrm{SU}(2)_{L}$ doublet $\Phi_{0}$ such that the kinetic terms in $\mathcal{L}_{H}$ have the customary unit normalization,

$$
H_{0}=Z_{\Phi}^{1 / 2} H, \quad \mathbf{w}_{0}=Z_{\Phi}^{1 / 2} \quad \mathbf{w}, \Phi_{0}=Z_{\Phi}^{1 / 2} \Phi,
$$

and to introduce a corresponding bare vacuum expectation value $v_{0}$, a bare coupling $\lambda_{0}$, and a parameter $\delta m_{0}^{2}$ :

$$
\begin{gathered}
v_{0}=Z_{\Phi}^{1 / 2} v \\
\lambda_{0}=\frac{\lambda}{Z_{\Phi}^{2}}\left(1+\frac{\delta \lambda}{\lambda}\right), \\
\delta m_{0}^{2}=Z_{\Phi}^{-1}\left[\mu^{2}+\delta \mu^{2}-(\lambda+\delta \lambda) v^{2}\right] .
\end{gathered}
$$

With these definitions, we obtain the form of $\mathcal{L}_{H}$ that we will use: 


$$
\begin{aligned}
\mathcal{L}_{H}= & \frac{1}{2}\left(\partial_{\mu} \mathbf{w}_{\mathbf{0}} \cdot \partial^{\mu} \mathbf{w}_{\mathbf{0}}+\partial_{\mu} H_{0} \partial^{\mu} H_{0}\right) \\
& -\frac{1}{4} \lambda_{0}\left(\mathbf{w}_{0}^{2}+H_{0}^{2}+2 v_{0} H_{0}\right)^{2} \\
& +\frac{1}{2} \delta m_{0}^{2}\left(\mathbf{w}_{0}^{2}+H_{0}^{2}+2 v_{0} H_{0}\right) .
\end{aligned}
$$

The fermion Lagrangian $\mathcal{L}_{F}$ can be treated in a similar fashion. There is a separate $\mathrm{SU}(2)_{L} \times \mathrm{U}(1)_{Y}$-symmetric counterterm for each term in Eq. (2.3). These counterterms can be absorbed in the definitions of a set of bare fields and couplings to bring $\mathcal{L}_{F}$ into the form

$$
\begin{aligned}
& \mathcal{L}_{F}=i \bar{\Psi}_{L}^{0} \not \Psi_{L}^{0}+i \bar{\psi}_{t, R}^{0} \partial \psi_{t, R}^{0}+i \bar{\psi}_{b, R}^{0} \theta \psi_{b, R}^{0}-\frac{1}{\sqrt{2}} g_{t}^{0} \bar{\Psi}_{L}^{0} \widetilde{\Phi}_{0} \psi_{t, R}^{0}-\frac{1}{\sqrt{2}} g_{b}^{0} \bar{\Psi}_{L}^{0} \Phi_{0} \psi_{b, R}^{0}+\text { H.c. } \\
& =i \overline{\psi_{t}^{0}} \not \psi_{t}^{0}-\left(m_{t}+\delta m_{t}\right) \overline{\psi_{t}^{0}} \psi_{t}^{0}-i \overline{\psi_{b}^{0}} \not \psi_{b}^{0}-\left(m_{b}+\delta m_{b}\right) \bar{\psi}_{b}^{0} \psi_{b}^{0}-\frac{1}{\sqrt{2}} g_{t}^{0} \overline{\psi_{t}^{0}} H_{0} \psi_{t}^{0}+\frac{i}{\sqrt{2}} g_{t}^{0} \overline{\psi_{t}^{0}} \gamma^{5} z_{0} \psi_{t}^{0}-\frac{1}{\sqrt{2}} g_{b}^{0} \overline{\psi_{b}^{0}} H_{0} \psi_{b}^{0} \\
& -\frac{i}{\sqrt{2}} g_{b}^{0} \bar{\psi}_{b}^{0} \gamma^{5} z_{0} \psi_{b}^{0}+g_{t}^{0} \bar{\psi}_{t, R}^{0} w_{0}^{+} \psi_{b, L}^{0}+g_{t}^{0} \overline{\psi_{b, L}^{0}} w_{0}^{-} \psi_{t, R}^{0}-g_{b}^{0} \bar{\psi}_{b, R}^{0} w_{0}^{+} \psi_{t, L}^{0}-g_{b}^{0} \overline{\psi_{t, L}^{0}} w_{0}^{-} \psi_{b, R}^{0}
\end{aligned}
$$

where

$$
\begin{gathered}
\Psi_{L}^{0}=Z_{L}^{1 / 2} \Psi_{L}, \\
\psi_{f, R}^{0}=Z_{f, R}^{1 / 2} \psi_{f, R}, \quad f=t, b, \\
g_{f}^{0}=\frac{g_{f}}{Z_{\Phi}^{1 / 2}}\left(1+\frac{\delta g_{f}}{g_{f}}\right) .
\end{gathered}
$$

The parameters $m_{f}$ and $g_{f}$ are defined to be the physical masses and Yukawa couplings of the quarks, while $\delta m_{f}$ is the mass or coupling counterterm:

$$
m_{f}=g_{f} \frac{v}{\sqrt{2}}, \quad \delta m_{f}=m_{f} \frac{\delta g_{f}}{g_{f}} .
$$

Note that there are separate renormalization constants for $\psi_{t, R}$ and $\psi_{b, R}$, and a single renormalization constant for the left-handed doublet $\Psi_{L}$ [17]. The factor $Z_{\Phi}^{-1 / 2}$ in $g_{f}^{0}$ has been introduced for later convenience.

\section{B. Renormalization}

The determination of the renormalization constants is discussed in Appendix A. We use on-mass-shell renormalization with all quantities calculated in terms of the bare fields. The couplings and renormalizations are defined so that $\mathcal{L}_{\mathrm{GBA}}$ reproduces the results of the $\mathrm{SM}$ in the limit $M_{H}^{2} \gg M_{W}^{2}$. We emphasize here that this is not automatic: It is essential that the $w^{ \pm}$and $z$ fields be renormalized at a momentum scale $p^{2}$ with $\left|p^{2}\right| \ll M_{H}^{2}$ [16], and that the couplings be defined in terms of physical standard-model quantities that have well-defined limits for vanishing gauge couplings, $g, g^{\prime} \rightarrow 0$. In particular, since the $w^{ \pm}$and $z$ bosons are guaranteed to be massless by the Goldstone theorem [18], we renormalize the $w_{0}^{ \pm}$and $z_{0}$ fields at $p^{2}=0$, while the Higgs field is renormalized at $p^{2}=M_{H}^{2}$ with $M_{H}$ the physical Higgs-boson mass. The quartic coupling $\lambda$ will be defined [19] to be given exactly by the relation

$$
\lambda=M_{H}^{2} / 2 v^{2}=G_{F} M_{H}^{2} / \sqrt{2},
$$

where $G_{F}$ is the Fermi constant obtained from the muon decay rate using the standard electromagnetic radiative corrections and $v$ is the physical vacuum expectation value, $v=2^{-1 / 4} G_{F}^{-1 / 2} \approx 246 \mathrm{GeV}$. This definition connects smoothly with Goldstone limit as shown in [19,20].

With the specifications above, we find that the wave function renormalization constants $Z_{w}, Z_{z}$, and $Z_{H}$, which relate the bare fields $w_{0}^{ \pm}, z_{0}$, and $H_{0}$ to the physical fields through the relations

$$
w_{0}^{ \pm}=Z_{w}^{1 / 2} w_{\text {phys }}^{ \pm}, \quad z_{0}=Z_{z}^{1 / 2} z_{\text {phys }}, \quad H_{0}=Z_{H}^{1 / 2} H_{\text {phys }},
$$

are given to arbitrary order in terms of the self-energy functions for the bare fields by

$$
\begin{aligned}
& \frac{1}{Z_{w}}=1-\left.\frac{d}{d p^{2}} \Pi_{w}^{0}\left(p^{2}\right)\right|_{p^{2}=0}, \\
& \frac{1}{Z_{z}}=1-\left.\frac{d}{d p^{2}} \Pi_{z}^{0}\left(p^{2}\right)\right|_{p^{2}=0}, \\
& \frac{1}{Z_{H}}=1-\left.\frac{d}{d p^{2}} \Pi_{H}^{0}\left(p^{2}\right)\right|_{p^{2}=M_{H}^{2}} .
\end{aligned}
$$

The single mass counterterm $\delta m_{0}^{2}$ in the Higgs Lagrangian, Eq. (2.13), is given by

$$
\delta m_{0}^{2}=\Pi_{w}^{0}(0)=\Pi_{z}^{0}(0) .
$$

In the absence of fermions, the self-energy functions $\Pi_{w}^{0}$ and $\Pi_{z}^{0}$ would be equal for all $p^{2}$ because of the $\mathrm{SO}(3)$ symmetry of $\mathcal{L}_{H}$. That symmetry is destroyed in $\mathcal{L}_{\mathrm{GBA}}$ by the fermions, e.g., top and bottom quarks with $m_{t} \neq m_{b}$. As a result, $\Pi_{w}^{0}$ and $\Pi_{z}^{0}$ differ away from the special point $p^{2}=0$ [21], their derivatives consequently differ at $p^{2}=0$, and $Z_{z} \neq Z_{w}$. Furthermore, $Z_{H} \neq Z_{w}$ [20]. Since the wave 
function renormalization constant $Z_{\Phi}$ introduced earlier is sufficient, along with the other renormalization constants, to absorb the divergences in the Higgs sector of the theory, $Z_{w}, Z_{z}$, and $Z_{H}$ can only differ from $Z_{\Phi}$ by finite multiplicative factors $\widetilde{Z}_{i}, Z_{i}=\widetilde{Z}_{i} Z_{\Phi}$. An argument of Barbieri et al. [13] based on the Ward identity for the charged weak current determines $Z_{\Phi}$ and shows that $Z_{\Phi}=Z_{w}$ and $\widetilde{Z}_{w}=1$ in the limit $g, g^{\prime} \rightarrow 0$. The same result can be extracted from [16]. This identification corresponds physically to the observation that the Fermi constant $G_{F}$, and therefore the vacuum expectation value $v=Z_{\Phi}^{-1 / 2} v_{0}$, is defined through chargedcurrent processes, i.e., muon decay and superallowed nuclear $\beta$ decays. These involve the $W$ rather than the $Z$ or $H$ bosons. The distinction is important: The results we will present for the decay $H \rightarrow f \bar{f}$ in the Goldstone-boson approximation agree with the limit of the SM results for large $M_{H}$ only with the choice $Z_{\Phi}=Z_{w}$, though a finite theory would be obtained using any of the $Z$ 's.

The renormalization constants in the fermion sector are determined by the fermion self-energy function

$$
\Sigma^{0}=p \Pi_{V}^{0}\left(p^{2}\right)+p \gamma^{5} \Pi_{A}^{0}\left(p^{2}\right)+m \Pi_{S}^{0}\left(p^{2}\right),
$$

related to the bare propagator by

$$
i S_{F}^{0}=\frac{i}{\not p-m_{f}-\Sigma_{f}^{0}-\delta m},
$$

where we have suppressed the quark label. The renormalization to arbitrary order is rather intricate because of the separate right- and left-handed contributions to the physical fermion fields and $\Sigma^{0}$. This is discussed in Appendix A. At the one-loop level of interest in our applications, the results simplify substantially and are consistent with the SM results of Böhm et al. [17]. In particular, we find that

$$
Z_{L}=1+\Pi_{V}^{0}\left(m^{2}\right)-\Pi_{A}^{0}\left(m^{2}\right)+2 m^{2} \Pi_{V}^{0 \prime}\left(m^{2}\right)+2 m^{2} \Pi_{S}^{0 \prime}\left(m^{2}\right),
$$

$Z_{R}=1+\Pi_{V}^{0}\left(m^{2}\right)+\Pi_{A}^{0}\left(m^{2}\right)+2 m^{2} \Pi_{V}^{0 \prime}\left(m^{2}\right)+2 m^{2} \Pi_{S}^{0 \prime}\left(m^{2}\right)$,

$$
\frac{\delta g}{g}=\frac{\delta m}{m}=-\Pi_{V}^{0}\left(m^{2}\right)-\Pi_{S}^{0}\left(m^{2}\right) .
$$

We note finally that physical $n$-point scattering amplitudes are given by the truncated Green's functions $\Gamma_{n}^{0}$ calculated in terms of the bare fields, multiplied by a factor $\left(Z_{R}^{1 / 2} P_{R}+Z_{L}^{1 / 2} P_{L}\right)$ and the appropriate spinor for each incoming fermion line, and by a factor $\left(Z_{R}^{1 / 2} P_{L}+Z_{L}^{1 / 2} P_{R}\right)$ and a conjugate spinor for each outgoing fermion line, where $P_{R}=\frac{1}{2}\left(1+\gamma^{5}\right)$ and $P_{L}=\frac{1}{2}\left(1-\gamma^{5}\right)$. These factors generalize the usual factors of $Z^{1 / 2}$ for standard Dirac fields to the case of chiral interactions.

\section{III. $H \rightarrow f \bar{f}$ TO ONE LOOP: THEORY}

In the present section, we will sketch the calculation of the one-loop corrections to the matrix element for the decay $H \rightarrow f \bar{f}$ using the Goldstone-boson approximation and compare this approximate result, valid for $M_{H} \gg M_{W}$ indepen-
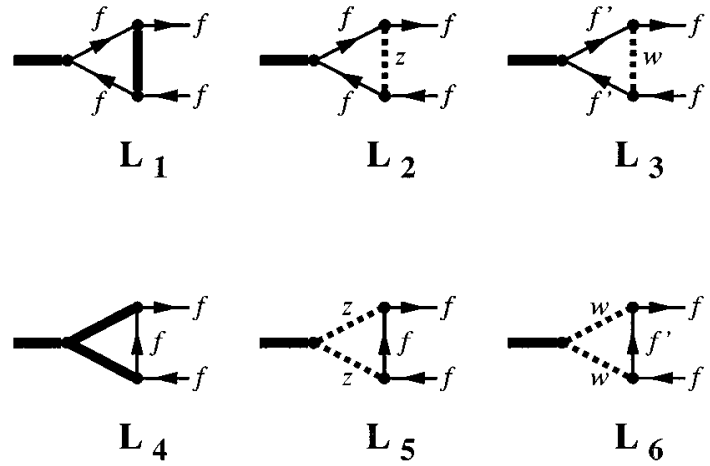

FIG. 1. The six triangle diagrams contributing to $H \rightarrow f \bar{f}$ at one loop within the framework of the Goldstone-boson approximation. Thick lines correspond to the Higgs particle, dotted lines represent the massless Goldstone bosons, and solid lines with arrows refer to fermions. The fermion $f^{\prime}$ is the $\mathrm{SU}(2)_{L}$ partner of $f$.

dently of the fermion masses, with the exact result obtained by other authors [22-25].

\section{A. Form of the decay matrix element}

According to the discussion above, the matrix element for the decay $H \rightarrow f \bar{f}$ is given by the expression

$$
\begin{aligned}
-i \mathcal{M}_{H \rightarrow f \bar{f}}= & Z_{H}^{1 / 2} \bar{u}\left(p_{1}-p_{2}, m_{f}\right)\left(Z_{R}^{1 / 2} P_{L}+Z_{L}^{1 / 2} P_{R}\right) \\
& \times \Gamma_{3}^{0}\left(Z_{R}^{1 / 2} P_{R}+Z_{L}^{1 / 2} P_{L}\right) v\left(p_{2}, m_{f}\right) .
\end{aligned}
$$

Here $p_{1}$ and $p_{2}$ are the momenta of the incoming Higgs boson and the outgoing antifermion, respectively, and $\Gamma_{3}^{0}$ is the truncated three-point vertex function calculated using the bare Lagrangian,

$$
\Gamma_{3}^{0}=-\frac{i}{\sqrt{2}} g_{f}^{0}+\sum_{i=1}^{6} L_{i}+\cdots,
$$

where the one-loop integrals $L_{i}$ correspond to the triangle diagrams in Fig. 1.

The renormalization constant $Z_{\Phi}$ appears only through the definition $g_{f}^{0}=Z_{\Phi}^{-1 / 2} g_{f}\left(1+\delta g_{f} / g_{f}\right)$, Eq. (2.17). Since $Z_{w}$ $\neq Z_{z} \neq Z_{H}$, it is essential to know which, if any, of the $Z_{i}$ 's should be used for $Z_{\Phi}$. As noted above, this can be established using the Ward identity for the weak vector current $[13,16]$, with the result $Z_{\Phi}=Z_{w}$ for our definition of the couplings and the $Z$ 's. Using the definition of $g_{f}^{0}$, writing each $Z$ as $1+\delta Z$, and expanding, we obtain the one-loop expression for $\mathcal{M}_{H \rightarrow f \bar{f}}$,

$$
\begin{aligned}
-i \mathcal{M}_{H \rightarrow f \bar{f}}= & \bar{u}\left(p_{1}-p_{2}, m_{f}\right) \\
& \times\left[-\frac{i}{\sqrt{2}} g_{f}\left(1+L_{\mathrm{bos}}+L_{\mathrm{fer}}\right)+\sum_{i=1}^{6} L_{i}\right] \\
& \times v\left(p_{2}, m_{f}\right),
\end{aligned}
$$

where the "bosonic" counterterm is

$$
L_{\mathrm{bos}}=\frac{Z_{H}^{1 / 2}}{Z_{w}^{1 / 2}}-1=\frac{1}{2} \delta Z_{H}-\frac{1}{2} \delta Z_{w}+\cdots
$$



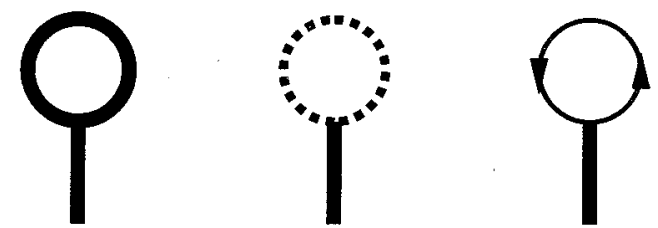

FIG. 2. The one-loop tadpole diagrams which are canceled by the counterterm $T_{0}$ to avoid a shift in the vacuum expectation value of the Higgs field. The different lines have the same meaning as in Fig. 1. A summation over all Goldstone boson and fermion loops is implied.

and the "fermionic" counterterm is

$L_{\mathrm{fer}}=\left(1+\frac{\delta g_{f}}{g_{f}}\right)\left(Z_{R}^{f} Z_{L}^{f}\right)^{1 / 2}-1=\frac{1}{2} \delta Z_{R}^{f}+\frac{1}{2} \delta Z_{L}^{f}+\frac{\delta g_{f}}{g_{f}}+\cdots$.

This grouping of terms has the advantage that $L_{\text {bos }}$, while dependent on the fermions through loop contributions, is independent of the flavor of the final fermion pair. It therefore gives a universal correction to all decays $H \rightarrow f \bar{f}$. The fermionic counterterm, in contrast, depends explicitly on the flavor of the final pair. This distinction will be important later.

\section{B. Bosonic counterterm}

The bosonic counterterm $L_{\text {bos }}$ defined in Eq. (3.4) is determined by the derivatives of the self-energy functions $\Pi_{w}^{0}$ and $\Pi_{H}^{0}$ in Eqs. (2.21) and (2.23). Because the field renormalization constant $Z_{H}$ differs from $Z_{w}=Z_{\Phi}$ only by a finite renormalization, the bosonic counterterm $L_{\text {bos }}$ is finite to all orders. The tadpole contributions to the self-energies shown in Fig. 2 are canceled by the same counterterm $T_{0}=v_{0} \delta m_{0}^{2}$ as cancels the apparent radiative changes in the physical vacuum expectation value $v$, and will be dropped. The remaining contributions to the boson self-energies are given to one loop by the irreducible diagrams shown in Figs. 3 and 4. The presence of fermion loops in these diagrams leads to a breaking of the $\mathrm{SO}(3)$ symmetry of the Higgs Lagrangian for unequal masses of the fermions in an $\mathrm{SU}(2)_{L}$ doublet, with the result that $\Pi_{z}^{0}\left(p^{2}\right) \neq \Pi_{w}\left(p^{2}\right)$ for $p^{2} \neq 0$, and hence that $Z_{z} \neq Z_{w}$.

The boson-loop diagrams have been calculated by a number of authors $[5-9,20,26,27]$. With the notation

$$
\delta Z_{i}=\delta Z_{i, \text { bos }}+\delta Z_{i, \text { fer }},
$$

the results are

$$
\begin{gathered}
\delta Z_{H, \text { bos }}=\frac{\lambda}{16 \pi^{2}}(12-2 \pi \sqrt{3}), \\
\delta Z_{w, \text { bos }}=\frac{\lambda}{16 \pi^{2}}(-1) .
\end{gathered}
$$

These one-loop quantities are separately finite. At two loops, the $Z$ 's become singular $[20,28,29]$, but the ratio $Z_{H, \text { bos }} / Z_{w, \text { bos }}$ and hence its contribution to $L_{\text {bos }}$ remain finite.
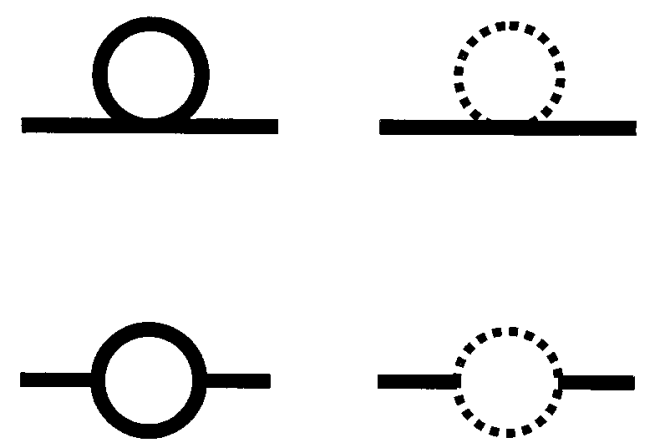

FIG. 3. Higgs self-energy contributions at one loop. The different lines have the same meaning as in Fig. 1. A summation over all Goldstone boson and fermion loops is implied.

The contributions $\delta Z_{H \text {,fer }}$ and $\delta Z_{w, \text { fer }}$ which arise from fermionic loops have been calculated in [30]. Neglecting all fermion masses except for $m_{t}$, the fermionic contribution to $L_{\text {bos }}$ is

$$
\begin{aligned}
\frac{1}{2} \delta Z_{H, \text { fer }}-\frac{1}{2} \delta Z_{w, \text { fer }} & \\
= & \frac{1}{16 \pi^{2}} N_{C} \frac{m_{t}^{2}}{v^{2}}\left[-\operatorname{Re}\left[B_{0}\left(M_{H}^{2}, m_{t}^{2}, m_{t}^{2}\right)-B_{0}\left(0,0, m_{t}^{2}\right)\right]\right. \\
& \left.-\left(1-\frac{1}{a^{2}}\right) M_{H}^{2} \operatorname{Re}\left[\partial B_{0}\left(M_{H}^{2}, m_{t}^{2}, m_{t}^{2}\right)\right]-\frac{1}{2}\right] .
\end{aligned}
$$

$N_{C}=3$ is the color factor. The standard scalar integral $B_{0}\left(p^{2}, m_{0}^{2}, m_{1}^{2}\right)$ and its derivative $\partial B_{0}\left(p^{2}, m_{0}^{2}, m_{1}^{2}\right)[27,31]$ are defined in Appendix B.
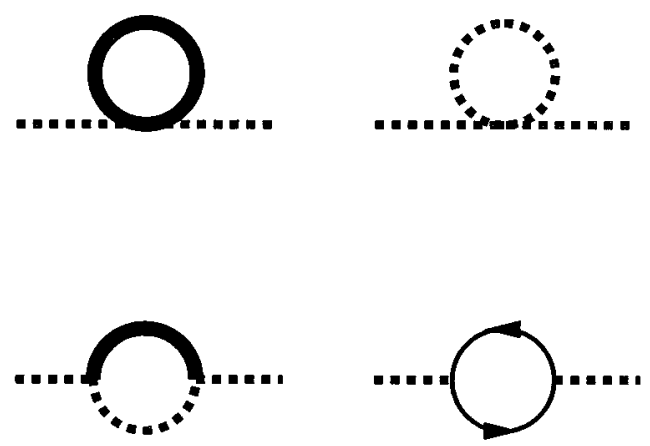

FIG. 4. The Goldstone-boson self-energies at one loop. The different lines have the same meaning as in Fig. 1. The diagrams on the right need to be summed over the different Goldstone-boson and fermion-loop contributions, respectively. In case of an external $w$ or $z$, the fermion loop consists of an $f f^{\prime}$ or $f f$ pair, respectively, where $f^{\prime}$ is the $\mathrm{SU}(2)_{L}$ partner of $f$. 
The explicit expression for $L_{\text {bos }}$ depends on the ratio of the Higgs-boson and top-quark masses. For Higgs-boson masses above the top-quark production threshold at $M_{H}=2 m_{t}$, we find that

$$
\begin{aligned}
L_{\text {bos }}= & \frac{\lambda}{16 \pi^{2}}\left(\frac{13}{2}-\pi \sqrt{3}\right) \\
& +\frac{3}{16 \pi^{2}} \frac{m_{t}^{2}}{v^{2}}\left\{b\left(2+\frac{1}{a^{2}}\right)\left[\ln 2 a+\ln \left(\frac{1+b}{2}\right)\right]\right. \\
& \left.-\frac{1}{2}-\frac{1}{a^{2}}\right\}, \quad M_{H}>2 m_{t},
\end{aligned}
$$

while, for smaller masses,

$$
\begin{gathered}
L_{\text {bos }}=\frac{\lambda}{16 \pi^{2}}\left(\frac{13}{2}-\pi \sqrt{3}\right)+\frac{3}{16 \pi^{2}} \frac{m_{t}^{2}}{v^{2}}\left[\frac{1}{2 a^{4}} \phi \sin (\phi)\right. \\
\left.-\left(2 a^{2}-3+\frac{1}{a^{2}}\right) \frac{\phi}{\sin (\phi)}-\frac{1}{2}-\frac{1}{a^{2}}\right] \\
M_{H}<2 m_{t},
\end{gathered}
$$

where

$$
\begin{gathered}
a=M_{H} / 2 m_{t}, \quad b=\sqrt{1-a^{-2}}, \\
\phi=\arccos \left(1-2 a^{2}\right), \quad 0<\phi<\pi .
\end{gathered}
$$

We will henceforth refer to the cases $M_{H}>2 m_{t}$ $\left(M_{H}<2 m_{t}\right)$ as those of a "heavy" ("light") Higgs boson.

As noted earlier, the result for $L_{\text {bos }}$ does not depend on the final state of the decay. For a fixed value of $M_{H}$, it is the same for all processes $H \rightarrow f \bar{f}$. As an useful example, we note that $L_{\text {bos }}$ already gives the complete one-loop $O(\lambda)$ and $O\left(g_{t}^{2}\right)$ corrections to the dominant leptonic decay, $H \rightarrow \tau^{+} \tau^{-}$. The fermionic counterterm and the vertex corrections for this and other leptonic decays do not receive further corrections in these couplings.

It is worth mentioning that there are no threshold singularities in $L_{\mathrm{bos}}$. The right-hand sides of Eqs. (3.10) and (3.11) are equal for $M_{H}=2 m_{t}$. In general, threshold singularities occur only when the gauge couplings of the standard model are included. We can consistently neglect the gauge couplings when using the Goldstone-boson approximation.

\section{Fermionic counterterm}

The fermionic counterterm $L_{\text {fer }}$ defined in Eq. (3.5) can easily be reduced to the form

$$
L_{\mathrm{fer}}=\operatorname{Re}\left\{\Pi_{S, f}^{0}\left(m_{f}^{2}\right)-2 m_{f}^{2}\left[\Pi_{V, f}^{0}\left(m_{f}^{2}\right)+\Pi_{S, f}^{0}\left(m_{f}^{2}\right)\right]\right\}
$$

by using the renormalization conditions stated in Eqs. (2.27), (2.28), and (2.29). The potential tadpole contributions to the fermionic self-energies and their derivatives are exactly canceled by the counterterms. The remaining one-particle irreducible diagrams are shown in Fig. 5. All involve loops con-
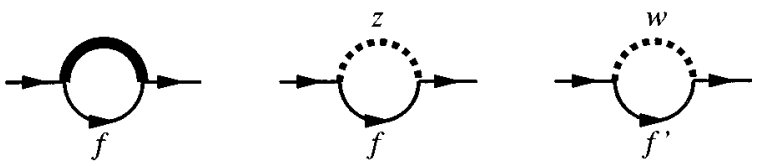

FIG. 5. The three diagrams contributing to the fermion selfenergies at one loop. The different lines have the same meaning as in Fig. 1. The fermion $f^{\prime}$ is the $\mathrm{SU}(2)_{L}$ partner of the external fermion $f$.

taining either a virtual Higgs boson or a massless Goldstone boson and the appropriate fermion line, $f$ or $f^{\prime}$, where $f$ refers to the final fermion.

In contrast to the expressions for the bosonic selfenergies, the expressions for the fermion self-energies depend on the Dirac matrices and, in particular, on $\gamma^{5}$. This $\gamma^{5}$ dependence raises the possibility of problems with dimensional regularization related to the definition of such quantities as $\operatorname{Tr} \gamma^{5} \gamma^{\mu_{1}} \ldots \gamma^{\mu_{D}}$ and the antisymmetric tensor in $D$ dimensions. However, as shown by Barbieri et al. [13], naive dimensional regularization with $\gamma^{5}$ and the remaining Dirac matrices treated as anticommuting is equivalent in the present context to the proper 't Hooft-Veltman scheme [31] to at least two loops for physical quantities. We have therefore calculated the fermion self-energies and the triangle diagrams using naive dimensional regularization.

The explicit evaluation of the expression in Eq. (3.13) requires the specification of the flavor of the final state fermion. The results for $f=t$ and $f=b$ are given in Secs. IV A and IV B, respectively.

\section{IV. $H \rightarrow f \bar{f}$ TO ONE LOOP: APPLICATIONS}

The six one-loop vertex diagrams $L_{i}, i=1-6$, contributing to Eq. (3.5) are shown in Fig. 1. Using the Dirac equations for the spinors $\bar{u}\left(p_{1}-p_{2}, m_{f}\right)$ and $v\left(p_{2}, m_{f}\right)$, extracting a factor $-i m_{f} / v$ from the reduced expressions for the functions $L_{i}$, and denoting the results by $\widetilde{L_{i}}$, we can write Eq. (3.3) as

$$
-i \mathcal{M}_{H \rightarrow f \bar{f}}=-i \frac{m_{f}}{v} \bar{u}\left(p_{1}-p_{2}, m_{f}\right) v\left(p_{2}, m_{f}\right)(1+\Delta \mathcal{T}),
$$

where the spinor matrix element is purely scalar and the quantity $\Delta \mathcal{T}$ is defined as

$$
\Delta \mathcal{T}=L_{\text {bos }}+L_{\text {fer }}+L_{\text {tri }}, \quad L_{\text {tri }}=\sum_{i=1}^{6} \widetilde{L_{i}}
$$

The correction to the decay width can then be written as

$$
\begin{aligned}
\Gamma(H \rightarrow f \bar{f})=\Gamma_{\mathrm{B}}(H \rightarrow f \bar{f})|1+\Delta \mathcal{T}|^{2} \\
\quad=\Gamma_{\mathrm{B}}(H \rightarrow f \bar{f})\left[1+2 \operatorname{Re} \Delta \mathcal{T}+O\left(\lambda^{2}, \lambda g_{f}^{2}, g_{f}^{4}\right)\right],
\end{aligned}
$$

where [32] 


$$
\Gamma_{B}(H \rightarrow f \bar{f})=\frac{N_{c} m_{f}^{2} M_{H}}{8 \pi v^{2}}\left(1-\frac{4 m_{f}^{2}}{M_{H}^{2}}\right)^{3 / 2}
$$

is the Born result. The function $\Sigma_{i} \widetilde{L_{i}}$ is rather complicated, and we will only give the results needed for the decays $H \rightarrow t \bar{t}$ and $H \rightarrow b \bar{b}$. They are listed in the following sections.

\section{A. $\boldsymbol{H} \rightarrow \boldsymbol{t} \overline{\boldsymbol{t}}$}

In the mass region $M_{H}>2 m_{t}$, the only important fermionic decay of the Higgs boson is the decay into a pair of top quarks, with a branching ratio of approximately 10\% [33]. Since $m_{t} \gg m_{b}$, we can calculate the decay rate for $H \rightarrow t \bar{t}$ using our general results for $H \rightarrow f \bar{f}$ evaluated in the limit $m_{b}=0$. Using the relation $\lambda=M_{H}^{2} /\left(2 v^{2}\right)$, we add the contributions from the six triangular loops and obtain

$$
\begin{aligned}
L_{\mathrm{tri}}= & -\frac{1}{16 \pi^{2}}\left(\frac{m_{t}}{v}\right)^{2} \frac{4 a^{4}}{a^{2}-1}\left[\left(1+\frac{2}{a^{2}}-\frac{1}{a^{4}}\right) m_{t}^{2} C_{0}\left(M_{H}, m_{t}, m_{t}, m_{t}, M_{H}\right)+\left(9-\frac{6}{a^{2}}\right) m_{t}^{2} C_{0}\left(M_{H}, m_{t}, M_{H}, M_{H}, m_{t}\right)\right. \\
& -m_{t}^{2} C_{0}\left(M_{H}, m_{t}, 0,0, m_{t}\right)-\frac{1}{2 a^{2}} m_{t}^{2} C_{0}\left(M_{H}, m_{t}, 0,0,0\right)+\left(-6+\frac{7}{a^{2}}\right) \ln 2 a-b\left(-6+\frac{2}{a^{2}}+\frac{1}{a^{4}}\right)\left(\ln 2 a+\ln \frac{1+b}{2}\right) \\
& \left.+\frac{\pi \sqrt{3}}{2 a^{2}}-\frac{i \pi}{2 a^{4}}\left(a^{2}-b\right)\right],
\end{aligned}
$$

where

$$
a=\frac{M_{H}}{2 m_{t}} \quad b=\sqrt{1-\frac{1}{a^{2}}}
$$

This result, based purely on the Lagrangian of the Higgs sector and the interaction of the Higgs and the Goldstone bosons with the fermion sector, is in complete agreement with the corresponding result ${ }^{1}$ given by Kniehl [22], which was calculated using the full electroweak Lagrangian, assuming $M_{H}^{2}>4 m_{t}^{2} \gg M_{W}^{2} \gg m_{b}^{2}$ and neglecting terms that are not enhanced by an inverse power of $M_{W}^{2}$. That procedure is equivalent to the usual power-counting arguments [3,4], taking the limit $g_{2} \rightarrow 0$, with $g_{2} / M_{W}=2 / v$. The agreement of the two results demonstrates the validity of the Goldstone-boson approximation to one loop for $H \rightarrow t \bar{t}$.

To compare the decay matrix element in our approximate calculation with that obtained in the full electroweak calculation, we still need explicit expressions for the counterterms $L_{\text {bos }}$ and $L_{\text {fer }}$ for $M_{H}>2 m_{t}$. The expression for $L_{\text {bos }}$ is given in Eq. (3.10). Evaluating the general expression for $L_{\text {fer }}$ for $M_{H}>2 m_{t}$ using $m_{b}=0$, we find that

$$
L_{\mathrm{fer}}=\frac{1}{16 \pi^{2}} \frac{m_{t}^{2}}{v^{2}}\left[\left(32 a^{4}-36 a^{2}+4\right) \ln 2 a-8 a^{2}+7 \frac{1}{16 \pi^{2}} \frac{m_{t}^{2}}{v^{2}}-b\left(32 a^{4}-20 a^{2}\right)\left(\ln 2 a+\ln \frac{1+b}{2}\right)\right] .
$$

$L_{\text {fer }}$ includes terms proportional to $a^{4} \ln 2 a, a^{4}, a^{2} \ln 2 a$, and $a^{2}$ which involve powers of $M_{H}^{2} / m_{t}^{2}$ and are enhanced for large Higgs-boson masses. However, these apparent enhancement terms cancel exactly when we expand the quantity $b$ in terms of $1 / a^{2}$; that is, $L_{\text {fer }}$ is actually not enhanced in powers of $M_{H}$. This is a manifestation of the Veltman screening theorem [34]: At one loop, an internal Higgs-boson line with a large mass $M_{H}$ leads only to a logarithmic enhancement. Since $L_{\text {fer }}$ is derived from two-point functions with external fermion lines, no powerlike enhancement is possible.

Assembling the partial results according to Eq. (4.2), we find the complete one-loop result for $\Delta \mathcal{T}$ in the limit $m_{t} \gg m_{f}$ for $f \neq t$,

$$
\begin{aligned}
\Delta \mathcal{T}= & -\frac{1}{16 \pi^{2}}\left(\frac{m_{t}}{v}\right)^{2}\left\{\frac { a ^ { 2 } } { a ^ { 2 } - 1 } \left[\left(1+\frac{2}{a^{2}}-\frac{1}{a^{4}}\right) M_{H}^{2} C_{0}\left(M_{H}, m_{t}, m_{t}, m_{t}, M_{H}\right)+\left(9-\frac{6}{a^{2}}\right) M_{H}^{2} C_{0}\left(M_{H}, m_{t}, M_{H}, M_{H}, m_{t}\right)\right.\right. \\
& \left.-M_{H}^{2} C_{0}\left(M_{H}, m_{t}, 0,0, m_{t}\right)-\frac{1}{2 a^{2}} M_{H}^{2} C_{0}\left(M_{H}, m_{t}, 0,0,0\right)+2 \pi \sqrt{3}-\frac{2 i \pi}{a^{2}}\left(a^{2}-b\right)\right]+8 a^{2}-\frac{11}{2}+\frac{3}{a^{2}} \\
& \left.+\left(-32 a^{4}+12 a^{2}+\frac{4}{a^{2}-1}\right) \ln (2 a)+b\left(32 a^{4}+4 a^{2}+10-\frac{3}{a^{2}}+\frac{12}{a^{2}-1}\right)\left(\ln (2 a)+\ln \frac{1+b}{2}\right)-2 a^{2}\left(\frac{13}{2}-\pi \sqrt{3}\right)\right\},
\end{aligned}
$$

\footnotetext{
${ }^{1}$ The result given in Eq. (4.5) should be compared with the quantity $(\alpha / 4 \pi) \delta_{\text {weak }}$ as defined by Kniehl.
} 


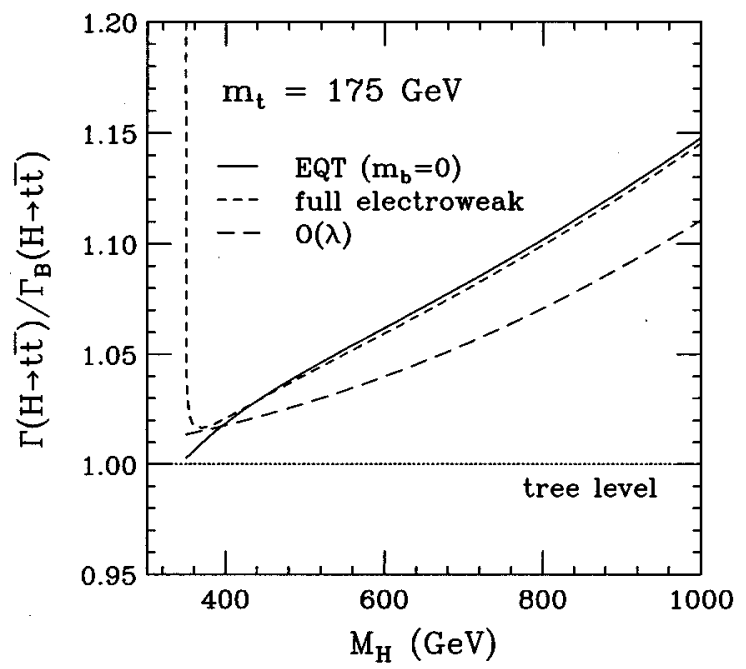

FIG. 6. The one-loop correction factor to the decay width $H \rightarrow t \bar{t}$. The solid curve gives the result of the Goldstone-boson approximation consisting of the sum of the $O(\lambda)$ and $O\left(g_{t}^{2}\right)$ corrections. Light fermion couplings are neglected. The result is compared with the full electroweak correction obtained in [22-24] and the GBA result without fermion corrections.

where we have replaced an overall factor $4 a^{2} m_{t}^{2}$ by $M_{H}^{2}$ in the coefficients of the $C_{0}$ functions. This result is exact in the context of the Goldstone-boson approximation except for the approximation $m_{f}=0, f \neq t$. The correction to the decay width is

$$
\Gamma(H \rightarrow t \bar{t})=\Gamma_{\mathrm{B}}(H \rightarrow t \bar{t})\left[1+2 \operatorname{Re} \Delta \mathcal{T}+O\left(\lambda^{2}, \lambda g_{t}^{2}, g_{t}^{4}\right)\right] .
$$

The functions $M_{H}^{2} C_{0}$ in Eq. (4.8) are functions only of the ratio $M_{H}^{2} / m_{t}^{2}$, i.e., of $a^{2}$. Expanding these functions for $a^{2} \gg 1$, we find that all of the contributions to $\Delta \mathcal{T}$ which are proportional to positive powers of $a^{2}$ cancel except for the last term in Eq. (4.8). That term arises from the boson-loop contribution to $L_{\text {bos }}$. The contributions from the fermion renormalization constants and the three-point functions are not power enhanced, but grow only as $\ln (2 a)=\ln \left(M_{H} / m_{t}\right)$. The purely bosonic corrections therefore give the dominant contribution to $\Delta \mathcal{T}$ for large Higgs-boson mass, a result that remains true to all orders in perturbation theory. In particular, at one loop,

$$
\begin{gathered}
\Delta \mathcal{T} \sim \frac{M_{H}^{2}}{16 \pi^{2} v^{2}}\left[\frac{1}{4}(13-2 \pi \sqrt{3})+O\left(\frac{m_{t}^{2}}{M_{H}^{2}} \ln \frac{M_{H}^{2}}{m_{t}^{2}}\right)+O\left(\frac{m_{t}^{2}}{M_{H}^{2}}\right)\right], \\
M_{H}^{2} \gg m_{t}^{2},
\end{gathered}
$$

and the fraction of the total correction associated with the top-quark Yukawa coupling decreases rapidly for $M_{H} \gg m_{t}$. However, the actual correction associated with $g_{t}$ may still be significant.

In Fig. 6 we show the approximate correction to the decay width, $\Gamma / \Gamma_{B}=1+2 \operatorname{Re} \Delta \mathcal{T}$, for $H \rightarrow t \bar{t}$ in the limit $m_{b}=0$ (solid curve). This result is compared with the full electroweak correction (short dashes) [35]. Away from the threshold, the Goldstone-boson approximation is excellent, giving a result which is only $3.9 \%(1.8 \%)$ larger in magni- tude than the full electroweak result for $M_{H}=500 \mathrm{GeV}$ (1 $\mathrm{TeV})$. This is roughly the accuracy one would expect when neglecting gauge contributions to $\Delta \mathcal{T}$ of order $\alpha / \pi$.

We find that the $O(\lambda)$ correction (long dashes) represents the dominant contribution to the GBA correction for all values of $M_{H}$ larger than $2 m_{t}$, i.e., above the decay threshold. The one-loop Yukawa correction $O\left(g_{t}^{2}\right)$ vanishes at about $M_{H}=400 \mathrm{GeV}$. For larger values of $M_{H}$, it is positive and adds to the $O(\lambda)$ contribution. While it initially grows more rapidly as a function of $M_{H}$ than the $O(\lambda)$ correction, the $M_{H}^{2}$ behavior of the latter wins over the asymptotically logarithmic growth of the Yukawa correction for $M_{H}$ greater than about $600 \mathrm{GeV}$. The Yukawa contribution represents a decreasing fraction of the total correction for higher masses, but is still significant numerically.

The very different behavior of the Goldstone-boson approximation and the full electroweak result at threshold is caused by a Coulomb singularity associated with the exchange of a virtual photon. Except for this QED effect, the GBA correction and the weak correction are in qualitative agreement at $M_{H}=2 m_{t}$ : The GBA correction is about zero at threshold, and the weak correction is of the order of $1 \%$. To have a better test of the validity of the GBA for values of $M_{H} \approx 2 m_{t}$, we next consider the decay $H \rightarrow b \bar{b}$ which is free of the Coulomb singularity at $M_{H}=2 m_{t}$.

\section{B. $H \rightarrow b \bar{b}$}

In the mass region of a "light" Higgs boson, $M_{H}<2 m_{t}$, the dominant fermionic decay of the Higgs boson is the (much suppressed) decay $H \rightarrow b \bar{b}$. We can still treat this decay using the Goldstone-boson approximation, provided that $M_{H}$ is large compared to the masses of the $W$ and $Z$ bosons, and can use the comparison of the results with those of the full electroweak theory to test the limits of validity of the approximation for a "light" Higgs boson. For a "heavy" Higgs boson, $M_{H}>2 m_{t}$, the approximate result and the full electroweak result would be expected to agree to an accuracy similar to that in the previous section. However, the dependence of the one-loop correction to $H \rightarrow b \bar{b}$ on $m_{t}$ is different from that encountered in top-quark production and therefore actually provides an independent check of the quality of the GBA for the case of a heavy Higgs boson.

We will again write the decay matrix element corrected to one loop in the form

$$
\begin{gathered}
-i \mathcal{M}_{H \rightarrow b \bar{b}}=-i \frac{m_{b}}{v} \bar{u}\left(p_{1}-p_{2}, m_{b}\right) v\left(p_{2}, m_{b}\right)(1+\Delta \mathcal{T}), \\
\Delta \mathcal{T}=L_{\mathrm{bos}}+L_{\mathrm{fer}}+L_{\mathrm{tri}} .
\end{gathered}
$$

The Born result is proportional to the ratio $m_{b} / v$. The oneloop GBA corrections contributing to $\Delta \mathcal{T}$ are proportional to $\lambda, m_{t}^{2} / v^{2}, m_{b}^{2} / v^{2}$, and squares of lighter fermion masses. Evaluating these contributions to $\Delta \mathcal{T}$, we set $m_{b}=0$ and also neglect other light fermions. In this limit, $L_{\text {fer }}$ only receives contributions from the bottom-quark self-energy diagram which contains a $(w, t)$ loop, Fig. 5 , and $L_{\text {tri }}$ reduces to a sum over only two triangle graphs, $L_{3}$ and $L_{6}$ in Fig. 1, with the tree-level factor $-i m_{b} / v$ extracted. 
The bosonic counterterm $L_{\text {bos }}$ is given by Eq. (3.10) or (3.11), depending on the value of $M_{H}$. The fermionic counterterm $L_{\text {fer }}$ and the triangle graphs $L_{\text {tri }}$ depend on the flavor of the final fermion pair and need to be reevaluated for Higgs-boson decay into bottom quarks. We find for $m_{b} \rightarrow 0$ that

$$
L_{\mathrm{fer}}=\frac{1}{16 \pi^{2}} \frac{m_{t}^{2}}{v^{2}}\left[-2 B_{0}\left(0,0, m_{t}^{2}\right)\right] .
$$

The triangle contribution is calculated from the expressions for $L_{3}$ and $L_{6}$ using the Dirac equation and setting $m_{b}=0$ :

$$
\begin{aligned}
L_{\mathrm{tri}}= & \frac{1}{16 \pi^{2}} \frac{m_{t}^{2}}{v^{2}}\left[2 B_{0}\left(0,0, m_{t}^{2}\right)+\left(2-\frac{1}{a^{2}}\right)\left[B_{0}\left(M_{H}^{2}, m_{t}^{2}, m_{t}^{2}\right)-B_{0}\left(0,0, m_{t}^{2}\right)\right]+2-4 \ln 2 a+\frac{1}{4 a^{4}} M_{H}^{2} C_{0}\left(M_{H}^{2}, 0, m_{t}^{2}, m_{t}^{2}, 0\right)\right. \\
& \left.+2\left(1+\frac{1}{4 a^{2}}\right) M_{H}^{2} C_{0}\left(M_{H}^{2}, 0,0,0, m_{t}^{2}\right)+2 i \pi\right] .
\end{aligned}
$$

The divergences related to the integrals $B_{0}$ cancel in the sum $\Delta \mathcal{T}=L_{\text {bos }}+L_{\text {fer }}+L_{\text {tri }}$. In case of a "light" Higgs, the complete one-loop electroweak radiative correction to the amplitude for the decay $H \rightarrow b \bar{b}$ is now found to be

$$
\begin{aligned}
\Delta \mathcal{T}= & \frac{1}{16 \pi^{2}} \frac{m_{t}^{2}}{v^{2}}\left[\frac{5}{2}-\frac{4}{a^{2}}-4 \ln 2 a-\left(\frac{1}{a^{2}}-\frac{2}{a^{4}}\right) \phi \sin (\phi)-3\left(2 a^{2}-3+\frac{1}{a^{2}}\right) \frac{\phi}{\sin (\phi)}+\frac{1}{4 a^{4}} M_{H}^{2} C_{0}\left(M_{H}^{2}, 0, m_{t}, m_{t}, 0\right)\right. \\
& \left.+2\left(1+\frac{1}{4 a^{2}}\right) M_{H}^{2} C_{0}\left(M_{H}^{2}, 0,0,0, m_{t}^{2}\right)+2 a^{2}\left(\frac{13}{2}-\pi \sqrt{3}\right)+2 i \pi\right], \quad M_{H}<2 m_{t},
\end{aligned}
$$

and the heavy-Higgs-boson result is

$$
\begin{aligned}
\Delta \mathcal{T}= & \frac{1}{16 \pi^{2}} \frac{m_{t}^{2}}{v^{2}}\left\{\frac{5}{2}-\frac{4}{a^{2}}-4 \ln 2 a+b\left(2+\frac{5}{a^{2}}\right)\left[\ln (2 a)+\ln \left(\frac{1+b}{2}\right)\right]+\frac{1}{4 a^{4}} M_{H}^{2} C_{0}\left(M_{H}^{2}, 0, m_{t}, m_{t}, 0\right)\right. \\
& \left.+2\left(1+\frac{1}{4 a^{2}}\right) M_{H}^{2} C_{0}\left(M_{H}^{2}, 0,0,0, m_{t}^{2}\right)+2 a^{2}\left(\frac{13}{2}-\pi \sqrt{3}\right)+i \pi\left[2-b\left(1+\frac{1}{a^{2}}\right)\right]\right\}, \quad M_{H}>2 m_{t} .
\end{aligned}
$$

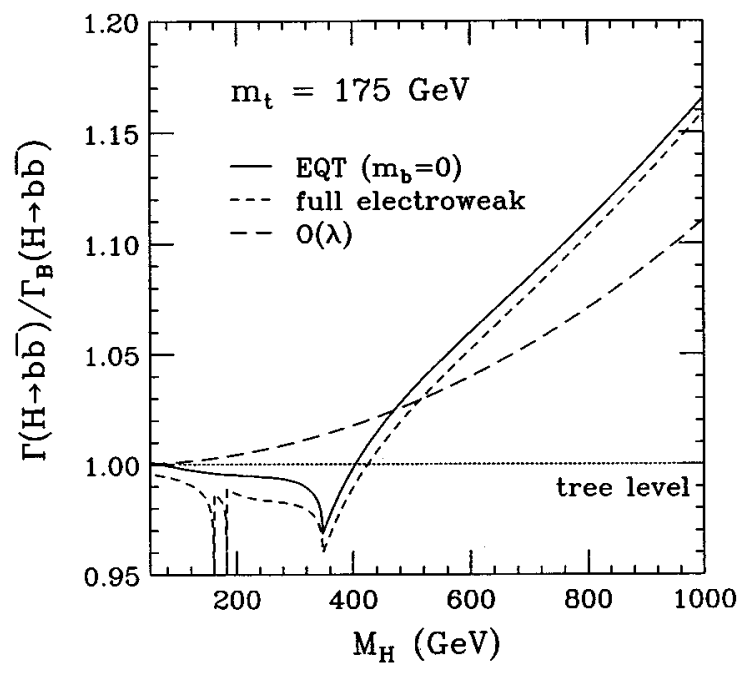

FIG. 7. The one-loop correction factor to the decay width $H \rightarrow b \bar{b}$. The solid curve gives to the result of the Goldstone-boson approximation, consisting of the sum of the $O(\lambda)$ and the $O\left(g_{t}^{2}\right)$ corrections. Light fermion couplings are neglected. The result is compared with the full electroweak correction obtained in [22-24] and the GBA result without fermion corrections.
In Fig. 7 we show the approximate correction to the decay width, $\Gamma / \Gamma_{B}=1+2 \operatorname{Re} \Delta \mathcal{T}$, for $H \rightarrow b \bar{b}$ in the limit $m_{b}=0$ (solid curve), and compare it with the full electroweak correction (short dashes) [35]. For large values of $M_{H}$, the approximation is very good, in agreement with our findings in the case of $H \rightarrow t \bar{t}$. The difference between the two corrections is again of the magnitude of the expected gauge corrections. The dominant contribution overall is the $O(\lambda)$ correction (long dashes), which grows more rapidly than the Yukawa correction for $M_{H}$ larger than about $700 \mathrm{GeV}$.

For a Higgs mass of about $400 \mathrm{GeV}$, the top-quark Yukawa correction cancels the $O(\lambda)$ contribution, and the one-loop radiative corrections are actually determined mainly by the gauge corrections. However, the magnitude of the gauge correction is still small compared to the magnitude of the Yukawa correction at this point, with an absolute value less than $1 \%$. The Goldstone-boson approximation breaks down for $M_{H}$ less than about $200 \mathrm{GeV}$, where the condition $M_{H} \gg M_{W}, M_{Z}$ is of questionable validity. The gauge corrections are the dominant corrections in this region and are larger in magnitude than both the Yukawa and the $O(\lambda)$ corrections. In addition, the gauge correction displays Coulomb singularities at $M_{H}=2 M_{Z}$ and $2 M_{W}$, a feature which is unique to gauge interactions and cannot be reproduced using Goldstone bosons and the limit $g, g^{\prime}=0$. This threshold region requires special treatment. 
Qualitatively, the GBA result approximates the full electroweak corrections rather well for Higgs masses larger than $200 \mathrm{GeV}$. The full electroweak correction remains finite at the threshold for top-quark production at $M_{H}=2 m_{t}$, and the expected threshold kink is reproduced by the approximate calculation. We conclude that the Goldstone-boson approximation is a useful tool even in the case of a "light" Higgs boson with a mass in the range $2 M_{Z}<M_{H}<2 m_{t}$, with the GBA correction to $\Gamma(H \rightarrow b \bar{b})$ giving a good estimate of the full electroweak correction.

\section{SUMMARY}

The equivalence theorem is known to be an excellent tool for describing heavy-Higgs-boson physics in the case of processes involving longitudinally polarized external gauge bosons $W_{L}^{ \pm} Z_{L}$ and Higgs scalars [1,2]. The closely related Goldstone-boson approximation provides a similarly simple method for calculating the dominant contributions of internal $W^{ \pm}, Z$, and $H$ bosons [3,4]. In either case, the vector bosons of the SM are replaced by the corresponding scalar Goldstone bosons in the symmetry-breaking sector of the theory, and the gauge couplings are ignored.

Most past applications of the GBA have neglected the Yukawa couplings of the fermions. This is not necessary; nor is it desirable. The top quark is quite massive [14]; $g_{t}$ is therefore large, and it is generally necessary to include topquark effects in calculations of electroweak radiative corrections to obtain accurate results. Because the approximations underlying the Goldstone-boson method are independent of the values of the Yukawa couplings, the method is easily extended to include fermions. However, it is necessary is to define the couplings and formulate the renormalization procedure in such a way that the reduced model is the limit of the SM for vanishing gauge couplings, with all quantities defined in terms of SM parameters that are well behaved in the limit.

In this paper, we have extended the usual Goldstoneboson approximation by systematically including the Yukawa interactions and formulating a renormalization procedure which is consistent with the requirements of the approximation, gives the correct relations to physical observables in the limit $g, g^{\prime} \rightarrow 0$, and is consistent with the powercounting arguments applied to the full SM $[3,4]$. The framework presented here permits quite simple calculations of the leading radiative corrections at arbitrary orders in $\lambda=M_{H}^{2} / 2 v^{2}$ and $g_{t}=\sqrt{2} m_{t} / v$. One can hope to obtain good approximate results for radiative corrections to Higgs-sector processes in the case of a "light" Higgs boson, $2 M_{Z}<M_{H}<2 m_{t}$, and excellent approximations for larger Higgs-boson masses.

As a test, we calculated the one-loop corrections to fermionic Higgs-boson decays using the Goldstone-boson approximation with fermions, and compared our results with the results obtained from a full electroweak calculation. Since the Yukawa interactions are negligible except for the top-quark coupling, we only included contributions from the latter. We find that the Higgs coupling $\lambda$ and the Yukawa coupling $g_{t}$ give the dominant corrections to the decay rates for $M_{H}>2 m_{t}$. The much smaller contributions of the transverse gauge couplings are only significant very close to 400
$\mathrm{GeV}$ in the case $H \rightarrow b \bar{b}$ where the dominant contributions cancel, and near the decay threshold for $H \rightarrow t \bar{t}$ where virtual photon exchange produces a Coulomb singularity.

In the mass range $2 M_{Z}<M_{H}<2 m_{t}$, the process $H \rightarrow b \bar{b}$ is the only significant fermionic decay. The one-loop radiative corrections to this decay associated with the quartic Higgs-boson coupling, the top-quark Yukawa coupling, and the transverse electroweak gauge couplings are all similar in magnitude, but with differing signs. In particular, a partial cancellation of the Higgs and Yukawa contributions makes the gauge correction equally important. The total correction is very small, less than $2 \%$. It seems plausible that the sum of the magnitudes of Higgs and Yukawa contributions would give a good estimate for an upper bound on the magnitude of the complete electroweak radiative correction for a variety of electroweak processes. In absence of cancellations between the $O\left(g_{t}^{2}\right)$ and $O(\lambda)$ corrections, the approximate result is expected to be the dominant correction for Higgs-boson masses larger than $2 M_{Z}$. Threshold singularities arising from the gauge sector cannot, of course, be reproduced using the Goldstone-boson approximation and require special treatment in any case.

According to Veltman's screening theorem [34], the $O(\lambda)$ corrections are the only one-loop corrections that grow proportionally to $M_{H}^{2}$. We find that this asymptotic growth of the correction is dominant only for Higgs-boson masses larger than $600-700 \mathrm{GeV}$, assuming a top-quark mass of 175 $\mathrm{GeV}$ [14]. For smaller values of $M_{H}$, we find the $O\left(g_{t}^{2}\right)$ corrections to have the stronger dependence on $M_{H}$.

In conclusion, we find that the calculation of radiative corrections using the GBA is greatly improved if the Yukawa interactions are included. The limit $m_{f}=0, f=t$ allows for a relatively simple calculation of the dominant radiative corrections, yielding an excellent approximation of the full electroweak corrections for the heavy-Higgs-boson case and order-of-magnitude estimates for $2 M_{Z}<M_{H}$ $<2 m_{t}$.

\section{ACKNOWLEDGMENTS}

The authors would like to thank B. Kniehl for providing the data for the full electroweak corrections shown in Figs. 6 and 7. One of the authors (K.R.) would like to thank A. Dabelstein for useful conversations and the Deutsche Forschungsgemeinschaft (DFG) for financial support under Contract No. Li519/2-1. The work of the other author (L.D.) was supported in part by the U.S. Department of Energy under Contract No. DE-FG02-95ER40896.

\section{APPENDIX A: RENORMALIZATION IN THE GOLDSTONE-BOSON APPROXIMATION}

As emphasized above, it is necessary to express the couplings in the reduced Lagrangian $\mathcal{L}_{\mathrm{GBA}}$ in terms of physical parameters of the standard model which have smooth limits for vanishing gauge couplings and to renormalize the theory in such a way that the connection with the SM is maintained. Several different approaches have been used in the renormalization of the SM as discussed by Böhm et al., and it is not especially straightforward to extract the limit of interest. We therefore give a systematic sketch of an appropriate all- 
orders renormalization procedure here. The renormalization in the Higgs sector has previously been discussed to arbitrary order by Maher et al. [20] using bare fields and by Ghinculov and van der Bij to two loops in the physical-fieldcounterterm formulation [28,29]. Both groups neglected the fermion contributions. The renormalization with fermions has apparently only been considered to one loop in earlier work [13].

\section{Fixing the renormalization of the Higgs Lagrangian}

The physical quartic coupling of the Higgs boson has been defined in Eq. (2.19), and the wave-function renormalization constants $Z_{w}, Z_{z}$, and $Z_{H}$ are defined in Eq. (2.20). The determination of the bare coupling $\lambda_{0}$ and the $Z$ 's proceeds as follows. The real part of the two-point function or inverse propagator $\Gamma^{(2)}\left(p^{2}\right)$ for each of the particles must vanish for $p^{2}$ equal to the square of the physical mass of that particle, $\Gamma^{(2)}\left(m^{2}\right)=0$. The two-point functions calculated using the bare fields are easily seen to have the form

$$
\begin{gathered}
\Gamma_{w_{0}}^{(2)}\left(p^{2}\right)=p^{2}-\Pi_{w}^{0}\left(p^{2}\right)+\delta m_{0}^{2}, \\
\Gamma_{z_{0}}^{(2)}\left(p^{2}\right)=p^{2}-\Pi_{z}^{0}\left(p^{2}\right)+\delta m_{0}^{2}, \\
\Gamma_{H_{0}}^{(2)}\left(p^{2}\right)=p^{2}-\Pi_{H}^{0}\left(p^{2}\right)-2 \lambda_{0} v_{0}^{2}+\delta m_{0}^{2},
\end{gathered}
$$

where the $\Pi$ 's are the bare self-energy functions. Since there is only a single mass counterterm $\delta m_{0}^{2}$ in the Higgs Lagrangian, Eq. (2.13), the vanishing of the renormalized masses $m_{w}$ and $m_{z}$ required by the Goldstone theorem leads to the relations

$$
\delta m_{0}^{2}=\Pi_{w}^{0}(0)=\Pi_{z}^{0}(0),
$$

and determines $\delta m_{0}^{2}$. The self-energy functions for the bare $w^{ \pm}$and $z$ fields must therefore be equal at $p^{2}=0$, an identity that holds to all orders in perturbation theory. In the following, we will replace $\delta m_{0}^{2}$ by $\Pi_{w}^{0}(0)$.

The condition that $v$ be the physical vacuum expectation value requires the vanishing of the truncated one-point function for the Higgs field,

$$
\Gamma_{H_{0}}^{(1)}(0)=-i T_{0}+i v_{0} \delta m_{0}^{2}=0
$$

where $T_{0}$ is the sum of all Higgs tadpole graphs (see Fig. 2) calculated using the bare fields. This gives the further relation $\delta m_{0}^{2}=T_{0} / v_{0}$, and so $\delta m_{0}^{2}$ can be calculated either as a self-energy or as a tadpole contribution. The resulting identity

$$
\Pi_{w}^{0}(0)=T_{0} / v_{0}
$$

provides a useful check on the calculations [36]. The vanishing of the one-point function, Eq. (A5), implies that the tadpole diagrams and the tadpole counterterm in Eq. (2.13) cancel order by order in the perturbation expansion and can be dropped together, as discussed by Taylor [37]. We have used this simplification throughout the paper.

The bare coupling $\lambda_{0}$ can be determined by using the definition $\lambda=M_{H}^{2} / 2 v^{2}$ and Eq. (A3). The on-mass-shell condition for the Higgs boson, $\Gamma_{H_{0}}^{(2)}\left(M_{H}^{2}\right)=0$ [38], gives the relation

$$
M_{H}^{2}=2 \lambda_{0} v_{0}+\Pi_{H}^{0}\left(M_{H}^{2}\right)-\delta m_{0}^{2} .
$$

Upon replacing $v_{0}^{2}$ by $Z_{\Phi} v^{2}=Z_{\Phi} M_{H}^{2} / 2 \lambda$ and $\delta m_{0}^{2}$ by $\Pi_{w}^{0}(0)$ in this expression and solving for $\lambda_{0}$, we find that

$$
\lambda_{0}=\lambda\left(1+\frac{\delta \lambda}{\lambda}\right) \frac{1}{Z_{\Phi}^{2}}=\frac{\lambda}{Z_{\Phi}}\left(1-\frac{\operatorname{Re} \Pi_{H}^{0}\left(M_{H}^{2}\right)-\Pi_{w}^{0}(0)}{M_{H}^{2}}\right) .
$$

Finally, the wave-function renormalization constants $Z_{w}, Z_{z}$, and $Z_{H}$, which relate the bare fields $w_{0}^{ \pm}, z_{0}$, and $H_{0}$ to the physical fields, are determined by the condition that the propagators for the physical fields have unit residue at the particle poles. The bare propagators do not, but instead have residues $Z_{i}$ given by

$$
Z_{i}=\left(\left.\frac{d}{d p^{2}} \Gamma_{i}^{(2)}\left(p^{2}\right)\right|_{p^{2}=m_{i}^{2}}\right)^{-1},
$$

a relation that leads directly to the results in Eq. (2.23).

The single wave-function renormalization constant $Z_{\Phi}$ necessary to remove the divergences in the theory can be identified using the Ward identities for the electroweak charged current which underlie both the equivalence theorem and the Goldstone-boson approximation [16,13]. For example, in their derivation of the equivalence theorem, Bagger and Schmidt [16] use the Ward identity to show that the ratio

$$
\frac{M_{W}^{0}}{M_{W}} \frac{Z_{W}^{1 / 2}}{Z_{w}^{1 / 2}}=\frac{g_{0} v_{0}}{g v} \frac{Z_{W}^{1 / 2}}{Z_{w}^{1 / 2}}=\frac{Z_{\Phi}^{1 / 2}}{Z_{w}^{1 / 2}}\left[1+O\left(g^{2}\right)\right]
$$

is unity up to corrections of order $g^{2}$ under conditions satisfied by our renormalization scheme. Thus, $Z_{\Phi}=Z_{w}$ in the limit $g, g^{\prime} \rightarrow 0$. Barbieri et al. [13] give a direct proof of this result.

\section{Fixing the renormalization of the fermion Lagrangian}

The bare self-energy functions and the bare propagator for the fermions are defined in Eqs. (2.25) and (2.26). Recalling the definition of the propagator, $i S_{F}(x-y)=$ $\langle\Omega|T(\psi(x), \bar{\psi}(y))| \Omega\rangle$, and the connection between the bare and physical fermion fields,

$$
\psi=\psi_{R}+\psi_{L}=\left(Z_{R}^{-1 / 2} P_{R}+Z_{L}^{-1 / 2} P_{L}\right) \psi^{0},
$$

we find that

$$
i S_{F}(p)=\left(Z_{R}^{-1 / 2} P_{R}+Z_{L}^{-1 / 2} P_{L}\right) i S_{F}^{0}(p)\left(Z_{R}^{-1 / 2} P_{L}+Z_{L}^{-1 / 2} P_{R}\right)
$$

or, calculating the inverse of $S_{F}^{0}$ explicitly, 


$$
\begin{aligned}
i S_{F}(p)= & \left(\not\left\{\frac{1}{2}\left(Z_{L}^{-1}+Z_{R}^{-1}\right)\left[1-\Pi_{V}^{0}\left(p^{2}\right)\right]-\frac{1}{2}\left(Z_{L}^{-1}-Z_{R}^{-1}\right) \Pi_{A}^{0}\left(p^{2}\right)\right\}\right. \\
& +\not p \gamma^{5}\left\{\frac{1}{2}\left(Z_{L}^{-1}-Z_{R}^{-1}\right)\left[1-\Pi_{V}^{0}\left(p^{2}\right)\right]-\frac{1}{2}\left(Z_{L}^{-1}+Z_{R}^{-1}\right) \Pi_{A}^{0}\left(p^{2}\right)\right\} \\
& \left.+Z_{L}^{-1 / 2} Z_{R}^{-1 / 2}\left[m+\delta m+m \Pi_{S}^{0}\left(p^{2}\right)\right]\right)\left(p^{2}\left\{\left[1-\Pi_{V}^{0}\left(p^{2}\right)\right]^{2}-\Pi_{A}^{02}\left(p^{2}\right)\right\}-\left(m+\delta m+m \Pi_{S}^{0}\right)^{2}\right)^{-1} .
\end{aligned}
$$

The mass or coupling counterterm $\delta m=m \delta g / g$ and the wave-function renormalization constants $Z_{R}$ and $Z_{L}$ are determined by the condition that the physical propagator describe a freely propagating particle with a mass $m$ and unit residue at the particle pole, $S_{F} \rightarrow 1 /(\not p-m)=(\not p+m) /\left(p^{2}\right.$ $-m^{2}$ ) for $p \rightarrow m . S_{F}$ has a simple pole at $p^{2}=m^{2}$ provided that [39]

$$
1+\frac{\delta m}{m}=1+\frac{\delta g}{g}=\sqrt{\left[1-\Pi_{V}^{0}\left(m^{2}\right)\right]^{2}-\Pi_{A}^{02}\left(m^{2}\right)}-\Pi_{S}^{0}\left(m^{2}\right) .
$$

The coefficient of $p \gamma^{5}$ in Eq. (A13) must vanish if $S_{F}$ is to be a normal massive propagator with equal right- and lefthanded residues at the particle pole. This requires that

$$
\left(Z_{R}-Z_{L}\right)\left[1-\Pi_{V}^{0}\left(m^{2}\right)\right]=\left(Z_{R}+Z_{L}\right) \Pi_{A}^{0}\left(m^{2}\right) .
$$

The coefficients of $p$ and $m$ in the numerator will be equal if, in addition,

$$
\begin{gathered}
\frac{1}{2}\left(Z_{R}+Z_{L}\right)\left[1-\Pi_{V}^{0}\left(m^{2}\right)\right]-\frac{1}{2}\left(Z_{R}-Z_{L}\right) \Pi_{A}^{0}\left(m^{2}\right) \\
=Z_{R}^{1 / 2} Z_{L}^{1 / 2}\left[1+\frac{\delta g}{g}+\Pi_{S}^{0}\left(m^{2}\right)\right] .
\end{gathered}
$$

Equations (A15) and (A16) are homogeneous of degree one in the $Z$ 's, and only determine the ratio $Z_{R} / Z_{L}$. The two equations may also be combined to obtain the pole condition Eq. (A14). The magnitude of the $Z$ 's is determined by the final condition that $S_{F}$ have unit residue at the pole. With the ratios $Z_{R} / Z_{L}$ determined as above for both the quarks in a doublet, this final condition can only be enforced for one of the two quark propagators by adjusting the single remaining renormalization constant $Z_{L}$ for the left-handed fields $\Psi_{L}$, Eq. (2.15). The second quark field requires an additional wave-function renormalization to reach the standard normalization. This extra renormalization constant is finite; the infinities in the wave-function renormalizations can be be absorbed entirely in $Z_{L}$ and the two $Z_{R}$ 's, which generate the only symmetric counterterms allowed in the kinetic part of $\mathcal{L}_{F}$, Eq. (2.14). Since our calculations are done entirely in terms of the bare fields, with the Z's appearing only in the overall factors necessary for external particles, the choice of definition for the original $Z_{L}$ is irrelevant, and we will give only the final renormalization constants. These are determined by setting the ratio of the coefficient of $\not p$ in Eq. (A13) to the derivative of the denominator equal to unity for $p^{2}=m^{2}$. Using Eqs. (A14)-(A16), we find that

$$
\begin{aligned}
\frac{1}{Z_{L}}= & 1-\Pi_{V}^{0}\left(m^{2}\right)+\Pi_{A}^{0}\left(m^{2}\right) \\
& -2 m^{2} \Pi_{S}^{0 \prime}\left(m^{2}\right) \sqrt{\frac{1-\Pi_{V}^{0}\left(m^{2}\right)+\Pi_{A}^{0}\left(m^{2}\right)}{1-\Pi_{V}^{0}\left(m^{2}\right)-\Pi_{A}^{0}\left(m^{2}\right)}} \\
& -2 m^{2} \frac{\left[1-\Pi_{V}^{0}\left(m^{2}\right)\right] \Pi_{V}^{0 \prime}\left(m^{2}\right)-\Pi_{A}^{0}\left(m^{2}\right) \Pi_{A}^{0 \prime}\left(m^{2}\right)}{1-\Pi_{V}^{0}\left(m^{2}\right)-\Pi_{A}^{0}\left(m^{2}\right)}
\end{aligned}
$$

and

$$
\begin{aligned}
\frac{1}{Z_{R}}= & 1-\Pi_{V}^{0}\left(m^{2}\right)-\Pi_{A}^{0}\left(m^{2}\right) \\
& -2 m^{2} \Pi_{S}^{0 \prime}\left(m^{2}\right) \sqrt{\frac{1-\Pi_{V}^{0}\left(m^{2}\right)-\Pi_{A}^{0}\left(m^{2}\right)}{1-\Pi_{V}^{0}\left(m^{2}\right)+\Pi_{A}^{0}\left(m^{2}\right)}} \\
& -2 m^{2} \frac{\left[1-\Pi_{V}^{0}\left(m^{2}\right)\right] \Pi_{V}^{0 \prime}\left(m^{2}\right)-\Pi_{A}^{0}\left(m^{2}\right) \Pi_{A}^{0 \prime}\left(m^{2}\right)}{1-\Pi_{V}^{0}\left(m^{2}\right)-\Pi_{A}^{0}\left(m^{2}\right)},
\end{aligned}
$$

where $\Pi^{\prime}\left(p^{2}\right)=d \Pi\left(p^{2}\right) / d p^{2}$. These expressions hold to all orders in perturbation theory, with one set for each quark. At the one-loop level of interest here, they simplify substantially. The expanded results are given in Eqs. (2.27), (2.28), and (2.29), and agree with those of Böhm et al. [17] obtained in the SM.

It is straightforward, finally, to establish the renormalization factors which must be used for the external fermions when scattering amplitudes are calculated using the bare fields. By using the standard reduction formulas [40], we can express the physical scattering amplitudes in terms of Fourier transforms of vacuum expectation values of time-ordered products of the physical fields. These vacuum expectation values appear multiplied by a factor $(\not p-m)$ and a spinor for each ingoing or outgoing fermion line. The physical fermion fields $\psi$ and $\bar{\psi}$ in the time-ordered products can be replaced by the bare fields $\psi^{0}$ and $\bar{\psi}^{0}$ using the definition in Eq. (A11). When the result is reexpressed in terms of the bare truncated Green's function $\Gamma_{n}^{0}$ the external factors are replaced by $S_{F}^{0}(p)\left(Z_{R}^{-1 / 2} P_{L}+Z_{L}^{-1 / 2} P_{R}\right)(\not p-m)$ and a spinor for each ingoing fermion line, and by $(p$ $-m)\left(Z_{R}^{-1 / 2} P_{R}+Z_{L}^{-1 / 2} P_{L}\right) S_{F}^{0}(p)$ and a conjugate spinor for each outgoing line. However, it follows from Eq. (A12) that

$$
\begin{aligned}
& S_{F}^{0}(p)\left(Z_{R}^{-1 / 2} P_{L}+Z_{L}^{-1 / 2} P_{R}\right)=\left(Z_{R}^{1 / 2} P_{R}+Z_{L}^{1 / 2} P_{L}\right) S_{F}(p), \\
& \left(Z_{R}^{-1 / 2} P_{R}+Z_{L}^{-1 / 2} P_{L}\right) S_{F}^{0}(p)=S_{F}(p)\left(Z_{R}^{1 / 2} P_{L}+Z_{L}^{1 / 2} P_{R}\right) .
\end{aligned}
$$


As a result, using the normalization of the physical propagator at $p=m$, we find that the physical scattering amplitudes are given by the truncated Green's functions $\Gamma_{n}^{0}$ calculated in terms of the bare fields, multiplied by a factor $\left(Z_{R}^{1 / 2} P_{R}+Z_{L}^{1 / 2} P_{L}\right)$ and the appropriate spinor for each incoming fermion line, and by a factor $\left(Z_{R}^{1 / 2} P_{L}+Z_{L}^{1 / 2} P_{R}\right)$ and a conjugate spinor for each outgoing fermion line.

\section{APPENDIX B: LOOP INTEGRAL EXPRESSIONS}

\section{Scalar integral expressions}

Here we define the scalar integral expressions needed to calculate the two- and three-point functions [27,31]. The more complicated vector and tensor loop integrals which arise from diagrams containing fermions can be reduced to sums of scalar integrals by standard techniques $[27,41]$. We will follow the definitions in [27].
For the calculation of the one-loop self-energies in $D$ dimensions we use

$$
A_{0}\left(m_{0}^{2}\right)=\frac{(2 \pi \mu)^{(4-D)}}{i \pi^{2}} \int d^{D} q \frac{1}{q^{2}-m_{0}^{2}+i \epsilon^{\prime}},
$$

$$
\begin{aligned}
B_{0}\left(p^{2}, m_{0}^{2}, m_{1}^{2}\right)= & \frac{(2 \pi \mu)^{(4-D)}}{i \pi^{2}} \int d^{D} q \\
& \times \frac{1}{\left[q^{2}-m_{0}^{2}+i \epsilon^{\prime}\right]\left[(q+p)^{2}-m_{1}^{2}+i \epsilon^{\prime}\right]} .
\end{aligned}
$$

The vertex corrections involve the integrals

$$
C_{0}\left(p_{1}^{2}, p_{2}^{2}, m_{0}^{2}, m_{1}^{2}, m_{2}^{2}\right)=\frac{(2 \pi \mu)^{(4-D)}}{i \pi^{2}} \int d^{D} q \frac{1}{\left[q^{2}-m_{0}^{2}+i \epsilon^{\prime}\right]\left[\left(q+p_{1}\right)^{2}-m_{1}^{2}+i \epsilon^{\prime}\right]\left[\left(q+p_{2}\right)^{2}-m_{2}^{2}+i \epsilon^{\prime}\right]}
$$

The arbitrary energy scale $\mu$ is introduced to fix the energy dimensions of the functions $A_{0}, B_{0}$, and $C_{0}$ independent of the value of $D$, and the infinitesimal quantity $i \epsilon^{\prime}$ defines the integration path in the complex plane. We note that Böhm et al. [17] define $A_{0}$ with an overall minus sign, while Kniehl $[22,42]$ introduces a minus sign in the definition of $C_{0}$.

These integrals can be evaluated in a straightforward manner using Feynman parameters. Complete analytic results are given by Denner [27], though it is useful to go back to the Feynman representation of the integrals for certain values of $p_{i}^{2}$ and $m_{i}^{2}$ to avoid problems with infrared singularities.

The integral $B_{1}$ that appears in the fermion self-energy functions is given by

$$
\begin{aligned}
B_{1}\left(p^{2}, m_{0}^{2}, m_{1}^{2}\right)= & \frac{1}{2 p^{2}}\left[A_{0}\left(m_{0}^{2}\right)-A_{0}\left(m_{1}^{2}\right)\right. \\
& \left.+\left(m_{1}^{2}-m_{0}^{2}-p^{2}\right) B_{0}\left(p^{2}, m_{0}^{2}, m_{1}^{2}\right)\right] .
\end{aligned}
$$

The derivatives of the self-energy functions with respect to $p^{2}$, the square of the external momentum, are needed in the calculation of the multiplicative wave-function renormalization constants $Z_{i} . A_{0}$ does not depend on the external momentum, and so does not contribute to the $Z$ 's. The derivatives

$$
\left.\partial B_{i}\left(M^{2}, m_{0}^{2}, m_{1}^{2}\right) \equiv \frac{\partial}{\partial p^{2}} B_{i}\left(p^{2}, m_{0}^{2}, m_{1}^{2}\right)\right|_{p^{2}=M^{2}}, \quad i=0,1,
$$

are given, for example, by Denner [27].

The reduction of the tensor integrals which appear in the fermion self-energy functions to sums of scalar integrals is discussed in detail in [27]. The results given there agree with our calculations [30] except for a typographical error in Denner's expression for the function $C_{00}$ in his Eq. (C.37). In our notation, the correct result for $C_{00}$ is

$$
\begin{aligned}
C_{00}= & \frac{1}{4}\left[B_{0}\left(\left(p_{1}-p_{2}\right)^{2}, m_{2}^{2}, m_{1}^{2}\right)+\left(m_{0}^{2}-m_{1}^{2}+p_{1}^{2}\right) C_{1}\right. \\
& \left.+\left(m_{0}^{2}-m_{2}^{2}+p_{2}^{2}\right) C_{2}+2 m_{0}^{2} C_{0}\right] .
\end{aligned}
$$

\section{APPENDIX C: TADPOLES AND SELF-ENERGY FUNCTIONS}

The only neutral field that receives a shift in its vacuum expectation value because of tadpole contributions is the Higgs field $h$; the $z$ field does not. The $z$ and $w$ fields have couplings through $\mathcal{L}_{H}$, Eq. (2.13), which require an even number of fields to participate, and no purely bosonic tadpole graphs can be formed for the $z$. With the addition of the fermionic Lagrangian $\mathcal{L}_{F}$, Eq. (2.14), the $z$ can form a tadpole with a fermion loop, but the presence of a factor $\gamma^{5}$ in the $z$-fermion coupling and a trace over the $\gamma$ matrices involved yields a vanishing result. However, fermion loops contribute to the Higgs tadpoles as shown in Fig. 2 since no $\gamma^{5}$ is involved in the $H$-fermion coupling. Taking into account both the bosonic and fermionic contributions, the Higgs one-point function (tadpole function) is

$$
T=\frac{1}{16 \pi^{2}}\left(-\frac{3 M_{H}^{2}}{2 v} A_{0}\left(M_{H}^{2}\right)+\sum_{f} N_{C}^{f} \frac{4 m_{f}^{2}}{v} A_{0}\left(m_{f}^{2}\right)\right) .
$$

The graphs of the one-loop one-particle irreducible selfenergy contributions to the bosonic and fermionic fields are shown in Figs. 3, 4, and 5. They can be evaluated as [27,30] 


$$
\begin{aligned}
\Pi_{H}^{0}\left(p^{2}\right)= & -\frac{1}{16 \pi^{2}}\left[3 \lambda A_{0}\left(M_{H}^{2}\right)+18 \lambda^{2} v^{2} B_{0}\left(p^{2}, M_{H}^{2}, M_{H}^{2}\right)\right. \\
& \left.-6 \lambda^{2} v^{2} B_{0}\left(p^{2}, 0,0\right)\right]+\frac{1}{16 \pi^{2}} \sum_{f} N_{C}^{f} \frac{m_{f}^{2}}{v^{2}} \\
& \times\left[4 A_{0}\left(m_{f}^{2}\right)-\left(2 p^{2}-8 m_{f}^{2}\right) B_{0}\left(p^{2}, m_{f}^{2}, m_{f}^{2}\right)\right], \\
\Pi_{z}^{0}\left(p^{2}\right)= & -\frac{1}{16 \pi^{2}}\left[\lambda A_{0}\left(M_{H}^{2}\right)+4 \lambda^{2} v^{2} B_{0}\left(p^{2}, M_{H}^{2}, 0\right)\right] \\
& +\frac{1}{16 \pi^{2}} \sum_{f} N_{C}^{f} \frac{m_{f}^{2}}{v^{2}} \\
& \times\left[4 A_{0}\left(m_{f}^{2}\right)-2 p^{2} B_{0}\left(p^{2}, m_{f}^{2}, m_{f}^{2}\right)\right], \\
\Pi_{w}^{0}\left(p^{2}\right)= & -\frac{1}{16 \pi^{2}}\left[\lambda A_{0}\left(M_{H}^{2}\right)+4 \lambda^{2} v^{2} B_{0}\left(p^{2}, M_{H}^{2}, 0\right)\right] \\
& -\frac{1}{16 \pi^{2}} \sum_{\left(f, f^{\prime}\right)} N_{C}^{f} \frac{1}{v^{2}}\left\{8 m_{f}^{2} m_{f^{\prime}}^{2} B_{0}\left(p^{2}, m_{f}^{2}, m_{f^{\prime}}^{2}\right)\right. \\
& +2\left(m_{f}^{2}+m_{f^{\prime}}^{2}\right)\left[-A_{0}\left(m_{f}^{2}\right)-A_{0}\left(m_{f^{\prime}}^{2}\right)\right. \\
& \left.\left.+\left(p^{2}-m_{f}^{2}-m_{f^{\prime}}^{2}\right) B_{0}\left(p^{2}, m_{f}^{2}, m_{f^{\prime}}^{2}\right)\right]\right\},
\end{aligned}
$$

$$
\begin{aligned}
\Pi_{V, f}^{0}\left(p^{2}\right)= & \frac{1}{16 \pi^{2}} \frac{m_{f}^{2}}{v^{2}}\left[-B_{1}\left(p^{2}, m_{f}^{2}, M_{H}^{2}\right)-B_{1}\left(p^{2}, m_{f}^{2}, 0\right)\right. \\
& \left.-\left(1+\frac{m_{f^{\prime}}^{2}}{m_{f}^{2}}\right) B_{1}\left(p^{2}, m_{f^{\prime}}^{2}, 0\right)\right], \\
\Pi_{A, f}^{0}\left(p^{2}\right)= & \frac{1}{16 \pi^{2}} \frac{m_{f}^{2}}{v^{2}}\left(-1+\frac{m_{f^{\prime}}^{2}}{m_{f}^{2}}\right) B_{1}\left(p^{2}, m_{f^{\prime}}^{2}, 0\right), \\
\Pi_{S, f}^{0}\left(p^{2}\right)= & \frac{1}{16 \pi^{2}} \frac{m_{f}^{2}}{v^{2}}\left(B_{0}\left(p^{2}, m_{f}^{2}, M_{H}^{2}\right)-B_{0}\left(p^{2}, m_{f}^{2}, 0\right)\right. \\
& \left.-2 \frac{m_{f^{\prime}}^{2}}{m_{f}^{2}} B_{0}\left(p^{2}, m_{f^{\prime}}^{2}, 0\right)\right) .
\end{aligned}
$$

The $z$ and $w$ self-energies only differ in the fermionic part, as expected. It is easily shown using the explicit results given for $A_{0}$ and $B_{0}$ in Appendix B that they are equal at one loop at $p^{2}=0$ independently of the values of the masses $m_{f}$ and $m_{f^{\prime}}$. This equality does not extend to the derivatives of the self-energy functions, and hence to the $Z$ 's.
[1] B. W. Lee, C. Quigg, and H. B. Thacker, Phys. Rev. Lett. 38, 883 (1977); Phys. Rev. D 16, 1519 (1977).

[2] M. S. Chanowitz and M. K. Gaillard, Nucl. Phys. B261, 379 (1985).

[3] H. Veltman, Phys. Rev. D 41, 2294 (1990).

[4] H.-J. He, Y.-P. Kuang, and X. Li, Phys. Rev. Lett. 69, 2619 (1992); Phys. Rev. D 49, 4842 (1994); H.-J. He, Y.-P. Kuang, and C.-P. Yuan, ibid. 51, 6463 (1995).

[5] G. Passarino, Phys. Lett. 156B, 231 (1985).

[6] W. J. Marciano and S. S. D. Willenbrock, Phys. Rev. D 37, 2509 (1988).

[7] L. Durand, J. M. Johnson, and J. L. Lopez, Phys. Rev. Lett. 64, 1215 (1990).

[8] G. Passarino, Nucl. Phys. B343, 31 (1990).

[9] L. Durand, J. M. Johnson, and J. L. Lopez, Phys. Rev. D 45, 3112 (1992).

[10] L. Durand, J. M. Johnson, and P. N. Maher, Phys. Rev. D 44, 127 (1991).

[11] L. Durand, P. N. Maher, and K. R. Riesselmann, Phys. Rev. D 48, 1084 (1993).

[12] A. Ghinculov, Nucl. Phys. B455, 21 (1995).

[13] R. Barbieri. M. Beccaria, P. Ciafaloni, G. Curci, and A. Viceré, Phys. Lett. B 288, 95 (1992); 312, 511(E) (1993); Nucl. Phys. B409, 105 (1993).

[14] CDF Collaboration, S. Leone, Report No. Fermilab-Conf-96207E, $m_{t}=175.6 \pm 5.7$ (stat) \pm 7.1 (syst) $\mathrm{GeV}$ (unpublished); M. Narain (for the D0 Collaboration), Report No. FermilabConf-96-192E, $m_{t}=170 \pm 15$ (stat) \pm 10 (syst) GeV (unpublished).
[15] J. Cornwall, D. Levin, and G. Tiktopoulos, Phys. Rev. D 10, 1145 (1974), Appendix A; C. E. Vayonakis, Lett. Nuovo Cimento 17, 383 (1976); G. Gounaris, R. Kögeler, and H. Neufeld, Phys. Rev. D 34, 3257 (1986); Y. Yao, and C. Yuan, ibid. 38, 2237 (1988).

[16] J. Bagger and C. Schmidt, Phys. Rev. D 41, 264 (1990); A. Aoki, Kyoto University Report No. RIFP-705, 1987 (unpublished); in Physics at TeV Scale, Proceedings of the Meeting, Tsukuba, Japan, 1988, edited by K. Hidaka and K. Hikasa (KEK, Tsukuba, 1988).

[17] M. Böhm, H. Spiesberger, and W. Hollik, Fortschr. Phys. 34, 687 (1986).

[18] J. Goldstone, Nuovo Cimento 54, 154 (1961); T.-P. Cheng and L.-F. Li, Gauge Theory of Elementary Particle Physics (Oxford University Press, Oxford, England, 1984), Chap. 5.

[19] A. Sirlin and R. Zucchini, Nucl. Phys. B266, 389 (1986).

[20] P. N. Maher, L. Durand, and K. Riesselmann, Phys. Rev. D 48, 1061 (1993); 52, 553(E) (1995).

[21] R. S. Lytel, Phys. Rev. D 22, 505 (1980).

[22] B. A. Kniehl, Nucl. Phys. B376, 3 (1992).

[23] A. Dabelstein and W. Hollik, Z. Phys. C 53, 507 (1992).

[24] D. Yu. Bardin, B. M. Vilenskiŭ, and P. Kh. Khristov, Yad. Fiz. 53, 240 (1991) [Sov. J. Nucl. Phys. 53, 152 (1991)].

[25] L. Durand, B. A. Kniehl, and K. Riesselmann, Phys. Rev. Lett. 72, 2534 (1994); 75, 1699(E) (1995).

[26] S. Dawson and S. Willenbrock, Phys. Rev. Lett. 62, 1232 (1989).

[27] A. Denner, Fortschr. Phys. 41, 307 (1993).

[28] A. Ghinculov and J. J. van der Bij, Nucl. Phys. B436, 30 (1995). 
[29] A. Ghinculov, Phys. Lett. B 337, 137 (1994); 346, 426(E) (1995).

[30] K. Riesselmann, University of Wisconsin thesis, 1994.

[31] G. 't Hooft and M. Veltman, Nucl. Phys. B153, 365 (1979).

[32] L. Resnick, M. K. Sundaresan, and P. J. S. Watson, Phys. Rev. D 8, 172 (1973).

[33] A. Mendez, Mod. Phys. Lett. A 5, 2087 (1990); 6, 1533(E) (1991).

[34] M. Veltman, Acta Phys. Pol. B 8, 475 (1977).

[35] For a comparison of the electroweak corrections with the QCD corrections see L. Durand, B. A. Kniehl, and K. Riesselmann, Phys. Rev. D 51, 5007 (1995).

[36] The two different approaches were used, for example, by Maher et al. [20] and by Ghinculov and van der Bij [28,29], respectively, in their calculations of the two-loop self energies.

[37] J. C. Taylor, Gauge Theories of Weak Interactions (Cambridge University Press, Cambridge, England, 1976), Chap. 14.

[38] We follow the convention for an unstable Higgs boson of equating $M_{H}^{2}$ with the value of $p^{2}$ at which the real part of the expression on the right-hand side of Eq. (A7) vanishes for $p^{2}=p^{2}+i \epsilon, \epsilon \rightarrow 0+. \delta m_{0}^{2}, v_{0}$, and $\lambda_{0}$ are taken as real. With this definition, $\Gamma_{H_{0}}^{(2)} \approx Z_{H}^{-1}\left(p^{2}-M_{H}^{2}+i M_{H} \Gamma_{H}\right)$ for $p^{2} \approx M_{H}^{2}$ with $\Gamma_{H}=-Z_{H} \operatorname{Im} \Pi_{H}^{0}\left(M_{H}^{2}\right)$. A definition of $M_{H}^{2}$ as the complex value of $p^{2}$ at which the expression in Eq. (A7) vanishes exactly (i.e., a pole mass in terms of the propagator) requires some changes which we will not discuss here.

[39] For an unstable fermion, we will use a definition analogous to that used for the Higgs boson [38], and define the square of the fermion mass as the vale of $p^{2}$ at which the real part of the denominator in Eq. (A13) vanishes. Other definitions are also possible.

[40] See, for example, J. D. Bjorken and S. D. Drell, Relativistic Quantum Fields (McGraw-Hill, New York, 1965), Chap. 16.

[41] G. Passarino and M. Veltman, Nucl. Phys. B160, 151 (1979), Appendixes D and E.

[42] B. A. Kniehl, Nucl. Phys. B352, 1 (1991), Appendix A. 